\title{
ATP2C1 gene mutations in Hailey-Hailey disease and possible roles of SPCA1 isoforms in membrane trafficking
}

\author{
M Micaroni ${ }^{*, 1}$, G Giacchetti ${ }^{2,3}$, R Plebani ${ }^{2,4}$, GG Xiao ${ }^{1}$ and L Federici ${ }^{2,4}$
}

\begin{abstract}
ATP2C1 gene codes for the secretory pathway $\mathrm{Ca}^{2+} / \mathrm{Mn}^{2+}$-ATPase pump type 1 (SPCA1) localizing at the golgi apparatus. Mutations on the human ATP2C1 gene, causing decreased levels of the SPCA1 expression, have been identified as the cause of the HaileyHailey disease, a rare skin disorder. In the last few years, several mutations have been described, and here we summarize how they are distributed along the gene and how missense mutations affect protein expression. SPCA1 is expressed in four different isoforms through alternative splicing of the ATP2C1 gene and none of these isoforms is differentially affected by any of these mutations. However, a better understanding of the tissue specific expression of the isoforms, their localization along the secretory pathway, their specific binding partners and the role of the C-terminal tail making isoforms different from each other, will be future goals of the research in this field.
\end{abstract}

Cell Death and Disease (2016) 7, e2259; doi:10.1038/cddis.2016.147; published online 9 June 2016

Facts

- Mutations occurring on the ATP2C1 gene clearly have no hotspots, although some mutations are redundant and the majority of missense mutations are spotted on specific exons.

- The C-terminal tails of two out of four SPCA1 isoforms display a sequence motif recognized by PDZ domains, potentially used to interact with different pools of protein and involved in different signaling pathways.

- SPCA1 has important roles in regulating membrane trafficking, not only as a $\mathrm{Ca}^{2+}$ pump able to trigger the $\mathrm{Ca}^{2+}$ influx into the lumen of the golgi apparatus (and of consequence the cytosolic peri-golgi $\mathrm{Ca}^{2+}$ concentration/ signaling), but it also has a direct role in organizing cargo maturation/delivery from the golgi apparatus, which is imbalanced in cancer and other diseases.

\section{Open Questions}

- Why mutations on the ATP2C1 gene cause a different etiology between human and mouse? Does the overlap of ATP2C1 gene with ASTE1 gene have a role in regulating the SPCA1 expression in a different manner between species?
- Identification of proteins interacting with the C-terminal tails of the SPCA1 isoforms, and their possible role in mediating the function and the sub-organellar redistribution of the different SPCA1 isoforms in different cell types.

- Although SPCA1 is ubiquitously expressed in all the tissues, why mutations occurring on the ATP2C1 gene are mostly affecting the skin?

The study of intracellular membrane trafficking is important for the understanding of cellular structure and organelle function, and the coordinated cellular activities within complex organisms. The intracellular transport can be divided into different phases, which include the synthesis of lipids and proteins in the endoplasmic reticulum (ER), their folding and quality control, transport from ER-to-golgi apparatus and across the golgi apparatus, and delivery of cargoes to their final destinations. The golgi apparatus also participates in the posttranslational modifications (mostly glycosylation) of many proteins and lipids during their transport, and it is the central station of the intracellular secretory pathway. ${ }^{1,2}$

The physiology of the secretory pathway and the golgi apparatus is finely regulated and maintained by pumps and channels that maintain the luminal $\mathrm{pH} /$ ion levels, making each sub-compartment of the golgi unique (i.e., the cis-side of the golgi apparatus is different from the trans-side). ${ }^{3}$ The secretory pathway $\mathrm{Ca}^{2+}$-ATPase pump type 1 (SPCA1) regulates the golgi luminal $\mathrm{Ca}^{2+}$ homeostasis, it is distributed

\footnotetext{
${ }^{1}$ School of Pharmaceutical Science and Technology, Dalian University of Technology, Dalian 116024, China; ${ }^{2}$ Aging Research Center (Ce.S.I.), University 'G. D'Annunzio' of Chieti-Pescara, Chieti 66100, Italy; ${ }^{3}$ Department of Neuroscience, Imaging and Clinical Sciences, University 'G. D’Annunzio' of Chieti-Pescara, Chieti 66100, Italy and ${ }^{4}$ Department of Medical Oral and Biotechnological Sciences, School of Medicine and Health Sciences, University 'G. D'Annunzio' of Chieti-Pescara, Chieti 66100, Italy *Corresponding author: M Micaroni, School of Pharmaceutical Science and Technology, Dalian University of Technology, West Campus, No. 2 Linggong Road, Dalian 116024, China. Fax: +86 4118498 6473; E-mail: massimo.micaroni@gmail.com

Abbreviations: CASQ, calsequestrin; COPI, coat protein complex I; HHD, Hailey-Hailey disease; IGF1R, insulin-like growth factor receptor; ER, endoplasmic reticulum; PMR1, the yeast homolog of SPCA1; SERCA, ER ATPase $\mathrm{Ca}^{2+}$ pump type 2; SPCA1, secretory pathway $\mathrm{Ca}^{2+}$-ATPase pump type 1; TGN, trans-Golgi network Received 20.11.15; revised 17.4.16; accepted 28.4.16; Edited by E Candi
} 
along the secretory pathway membranes ${ }^{4}$ and ubiquitously expressed in all tissues. ${ }^{5}$

The human ATP2C1 gene encoding for SPCA1 is located on chromosome $3 q 21$ and consists of 28 exons. ${ }^{6,7}$ Alternative processing at the 3 '-end of the human ATP2C1 pre-mRNA produces four distinct ATP2C1 splice variants (corresponding to SPCA1a-d proteins; Figure 1a), namely (i) SPCA1a from the splicing of exon 26 to exon 27 with the translation stop codon located in exon 27 producing a protein of 919 amino acids; (ii) SPCA1b which contains 939 amino acids and results from splicing of exons 27 to 28 following activation of the internal 5'-splice donor site D1; (iii) splicing of exons 26-28 gives rise to SPCA1c, which has 888 amino acids; (iv) splicing at internal site D2 in exon 27 to exon 28 gives rise to SPCA1d, which is the largest variant with 949 amino acids (Figure 1a').

The resulting four SPCA1 protein isoforms differ in their C-terminal cytosolic tails and are organized in actuator domain $(A)$, phosphorylation domain $(P)$, nucleotide-binding domain (N), 5 stalk helices (S) in the cytoplasm, and 10 transmembrane helices (M) (Figure 1b). ${ }^{8}$ The C-terminal tails of each SPCA1 isoforms, unique to each alternatively spliced product (Figure 1c), present characteristics for potential specific functions that we will discuss later. The alternative splicing is not present in other species where we have a single SPCA1 (Figure 1c).

A schematic representation of the ATP2C1 gene sequence and the relative encoded SPCA1 protein sequences is reported in Figure 2. Here, we show where the exons start/ finish (codons highlighted in yellow), which is the corresponding cytosolic (purple), transmembrane (gray) and luminal (blue) portion of SPCA1, as well as the C-terminal tail (azure).

\section{Hailey-Hailey Disease}

Mutations of the ATP2C1 gene have been reported in HaileyHailey disease (HHD) patients, ${ }^{6,7}$ originally described by the Hailey brothers (Hugh Edward and William Howard) in $1939 .{ }^{9}$ $\mathrm{HHD}$ is also known as familial benign chronic pemphigus ${ }^{10}$ or familial benign pemphigus. ${ }^{11}$ The prevalence of HHD is estimated to be $1: 50000 .^{12} \mathrm{HHD}$ is a monoallelic genetic disorder inherited in an autosomal dominant pattern, ${ }^{6,7}$ meaning that one copy of the altered gene is sufficient to cause the disorder. Comparison between genotype and phenotype failed to show a clear correlation between the nature of the mutation and the clinical features of HHD (age of onset, severity, progression). Extensive inter-familial and intrafamilial variation was noted in clinical features, as well as between families sharing the same mutation. ${ }^{13}$ Mutations in the ATP2C1 gene reduce the amount of functional SPCA1. This abnormality impairs cells ability to store $\mathrm{Ca}^{2+}$ normally. For unknown reasons, this abnormal $\mathrm{Ca}^{2+}$ storage affects keratinocytes more than other cells types. The abnormal regulation of $\mathrm{Ca}^{2+}$ impairs many cell functions, including cell adhesion. As a result, keratinocytes do not stick tightly to one another, which causes the epidermis to become fragile and less resistant to minor trauma. Because the skin is easily damaged, it develops raw, blistered areas, particularly in skin folds where there is moisture and friction.

Recent observations shed new light on a possible role for altered $\mathrm{Ca}^{2+}$ homeostasis and responsiveness in $\mathrm{HHD}$ keratinocytes in creating an early defect in differentiation process due to a reduced production of involucrin. ${ }^{14}$ Keratin expression was also delayed in acantholytic epidermal segments of HHD skin. ${ }^{15}$ Because of decreased $\mathrm{Ca}^{2+}$ stored in the lumen of the golgi apparatus of HHD keratinocytes, involucrin mRNA degraded in response to abnormally raised cytosolic $\mathrm{Ca}^{2+}$. ${ }^{16}$ From dermatological perspectives, the HHD patients have been treated with topical steroid preparations to help outbreaks. Several drugs (antibiotics, antifungals, corticosteroids, etc.) keep under control the progression of the disease but are ineffective for severe chronic or relapsing forms. Some HHD patients found relief in laser resurfacing that burns off the top layer of the epidermis, allowing healthy nonaffected skin to regrow in its place.

\section{Mutations on the Human ATP2C1 Gene}

Here, we review the literature about the mutations occurring on the ATP2C1 gene, and give a comprehensive view of where these mutations fall along the gene sequence. Furthermore, we focus our attention on why some missense mutations affect SPCA1 efficiency without affecting the protein levels and address our interest to amino acidic residues that could probably have a role in the correct functioning of the protein and are not yet fully characterized. Finally, we focus our attention on the possible role of three different cytosolic tails of the SPCA1a, SPCA1b, and SPCA1d isoforms, and their potential for binding different partners.

Several mutations have been reported on the ATP2C1 gene in HHD patients, some of which were redundant even if no evident hotspots have been recognized so far. In Table 1 we summarize all the mutations reported so far, mentioning their localization along the gene sequence, the kind of mutation as well as the resultant change in the amino acid sequence. All the mutations generating truncated protein, which supposedly do not have a complete functionality or precise sub-organellar localization or are destabilized and therefore arguably destined for degradation, are indicated as premature termination codon (PTC).

After careful reviewing of literature using PubMed and the Chinese Biological Medicine Database (http://www.sinomed. ac.cn/zh/), we listed a total of 166 unique mutations on the ATP2C1 gene that have been reported in HHD to date. Of them, $\sim 55 \%$ lead to PTC, supporting the possibility that haploinsufficiency of $A T P 2 C 1$ is a prevalent mechanism for the dominant inheritance of HHD. Twenty-four ( 14\%) were nonsense mutations, fifty-nine $(\sim 36 \%)$ were deletion/insertion mutations (of which five $(\sim 4 \%)$ are in-frame deletion and insertion mutations), thirty-four ( 20\%) were splice-site mutations, and forty-nine $(\sim 30 \%)$ were missense mutations (Table 1). Thus, many mutations predict the absence, or a marked reduction of the mutated ATP2C1 product via nonsense-mediated mRNA decay. Non-conservative amino acid changes in functional domains of the molecule are highly conserved between golgi $\mathrm{Ca}^{2+}$ pumps from different species, and between other $\mathrm{Ca}^{2+}$-ATPases. In fact, some of these mutations occur at amino acid residues conserved between SPCA1 and SERCA1 (the ER ATPase $\mathrm{Ca}^{2+}$ pump type 1); the latter has previously been studied by site-directed 
a

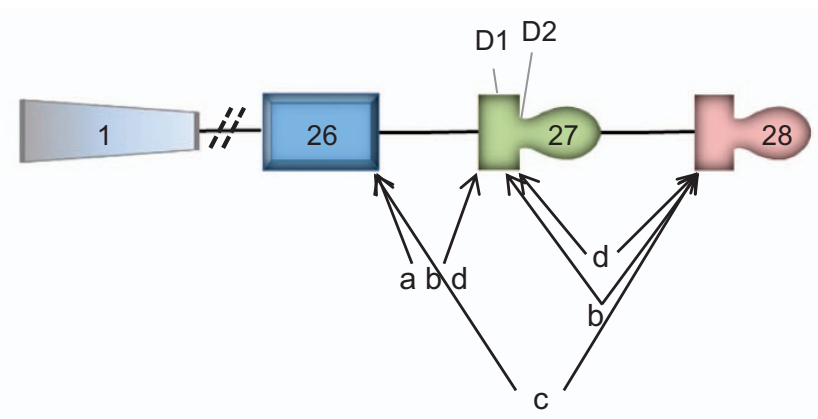

a'

a
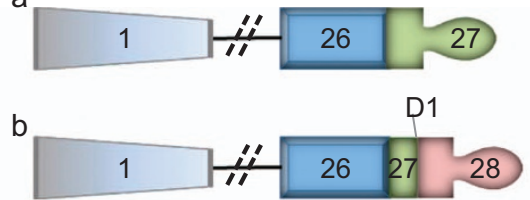

C

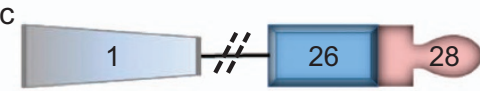

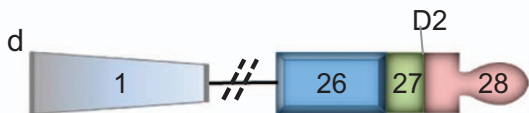

b

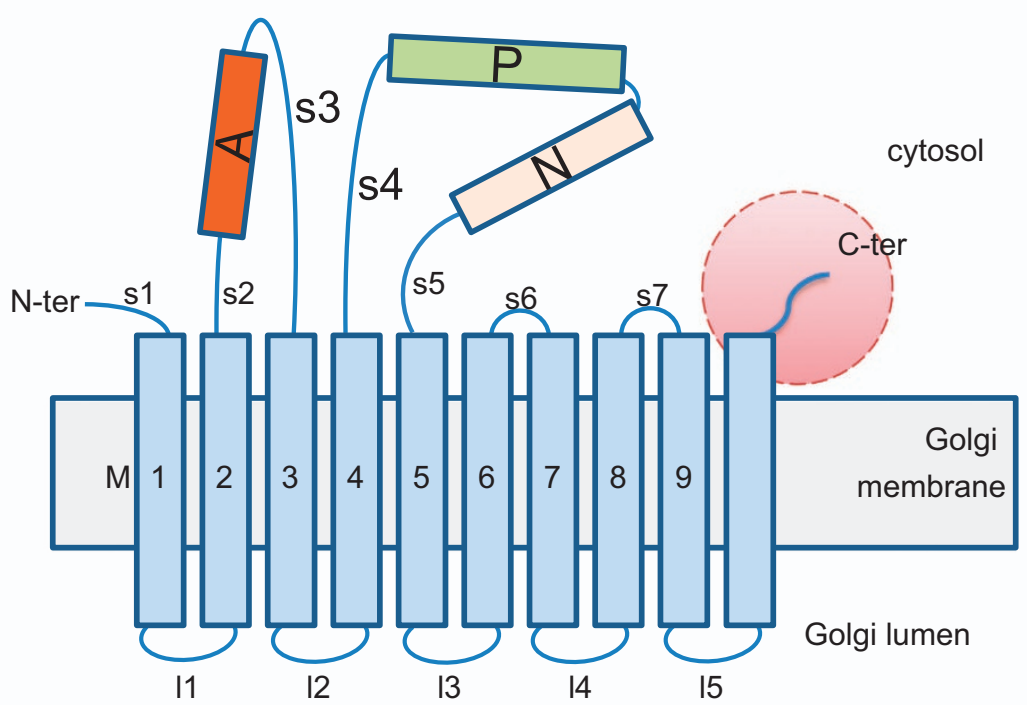

C

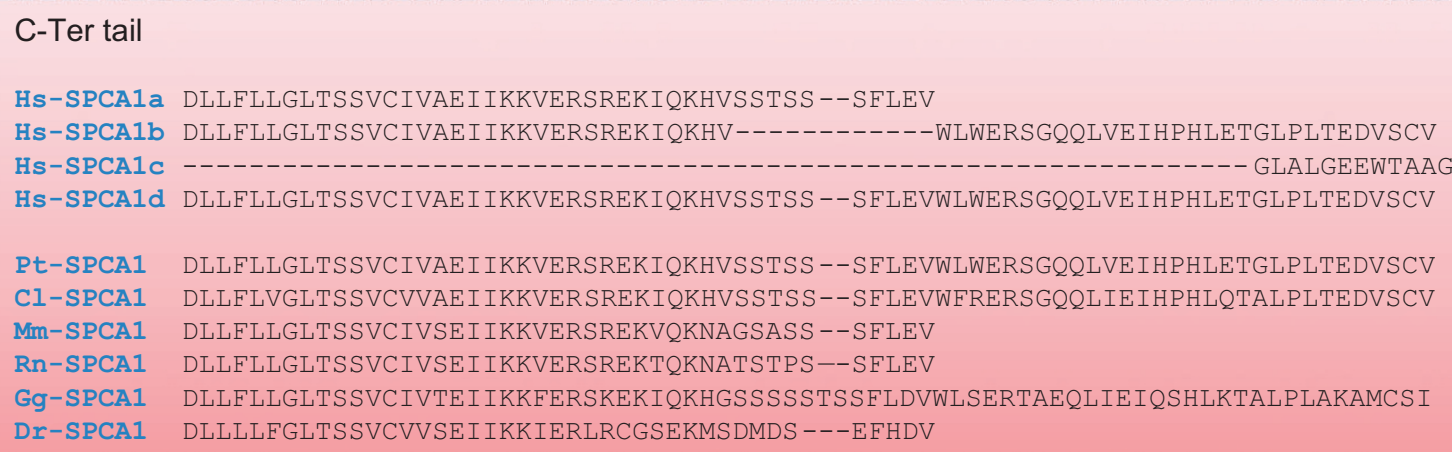

Figure 1 Representation of the ATP2C1 gene alternatively spliced and the molecular structure of encoded SPCA1. (a) The ATP2C1 gene consists of twenty-eight exons (represented by boxes), which are alternatively spliced as indicate by the internal $5^{\prime}$ donor splice sites, D1 and D2 generating four different mRNA. Diagonal lines illustrate the slicing patterns generating splice variants ATP2C1a-d. (a') The ATP2C1a-d splice variants are schematically represented. (a) and (a') are modified from Micaroni and Malquori. ${ }^{50}$ (b) Actuator domain (A), phosphorylation domain $(\mathrm{P})$, nucleotide-binding domain $(\mathrm{N})$ and 5 stalk helices $(\mathrm{S})$ in the cytoplasm, and 10 transmembrane helices (M). This figure was adapted from Matsuda et al. ${ }^{8}$ (c) In gray is the exon 26, in yellow the exon 27, in green the exon 28. According to the present literature, the isoform SPCA1c seems not be coded in a protein. This isoform is missing the exon 27 coding for the transmembrane 10 (M10). Furthermore, this isoform is missing the possibility to have a cytosolic C-terminal tail where potential binding sites for other proteins is present, reinforcing the idea that this isoform is not functional. Hs-SPCA1a (NP_055197); Hs-SPCA1b (NP_001001487.1); Hs-SPCA1c (NP_001001485.2); Hs-SPCA1d (NP_001001486.1); Pt-SPCA1 (XP_001145788.1); Cl-SPCA1 (XP_534262.2); Mm-SPCA1 (NP_778190.3); Rn-SPCA1 (NP_571982.2); Gg-SPCA1 (XP_015137243.1); Dr-SPCA1 (XP_003200287). Hs: Homo sapiens; Pt: Pan troglodytes; Cl: Canis lupus; Mm: Mus musculus; Rt: Rattus norvegicus; Gg: Gallus gallus; Dr: Danio rerio 
mutagenesis and the relevant mutations were shown to abrogate SERCA1 function. ${ }^{17}$

Mutations are scattered along the ATP2C1 gene without apparent clustering, showing a substantial allelic heterogeneity, and are distributed all over the encoded sequence (Supplementary Figure 1), as well as in the intron splice sites generating alternative splicing and/or truncated proteins. Mutations affect all domains of the resulting protein. Rarely, mutations seem to

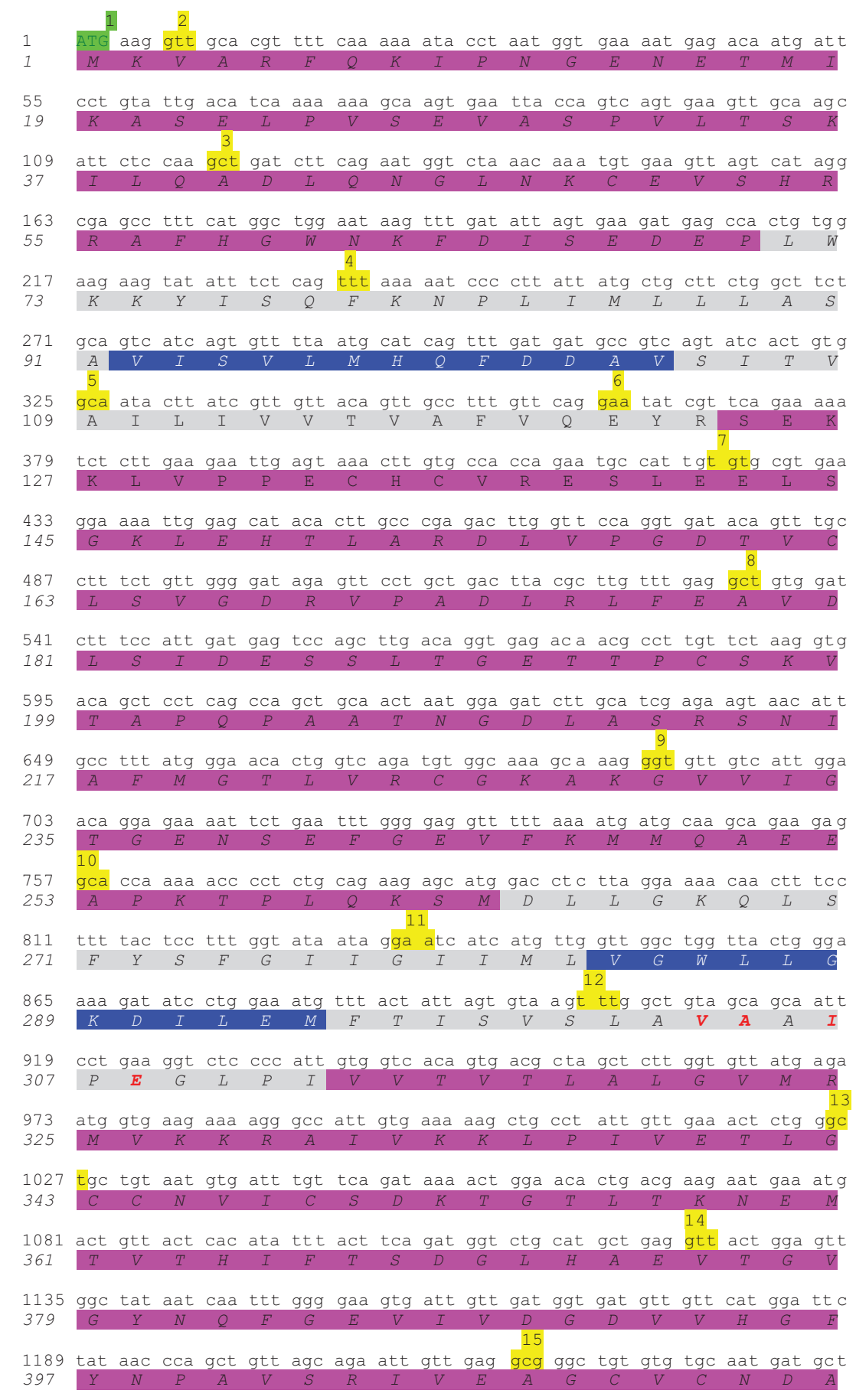

Figure 2 Identification of transmembrane domains vs cytosolic or luminal domains of SPCA1. For simplicity, SPCA1a (NP_055197) has been represented; other isoforms have identical sequence until the $\mathrm{C}$-terminal tail. The highlighted codons in green and in red are the starting and stop codon, respectively. Highlighted in yellow is the first codon of each corresponding numbered exon. Highlighted in gray is the transmembrane domain. The highlighted amino acids in purple are cytoplasmic, whereas those highlighted in blue are luminal. The highlighted amino acids in azure represent the cytosolic C-terminal tail (underlined is the KXD/E motif putative COPI-binding site). The bold typed red and blue amino acids are the residues directly involved in the binding of $\mathrm{Ca}^{2+} / \mathrm{Mn}^{2+}$ and $\mathrm{Mg}^{2+}$, respectively. Of note, the putative COPI-binding motif $\mathrm{KXD} / \mathrm{E}$ is just below the $\mathrm{M} 10$ domain. The prediction of these domains/residues was solved by similarity (UniProt database) 


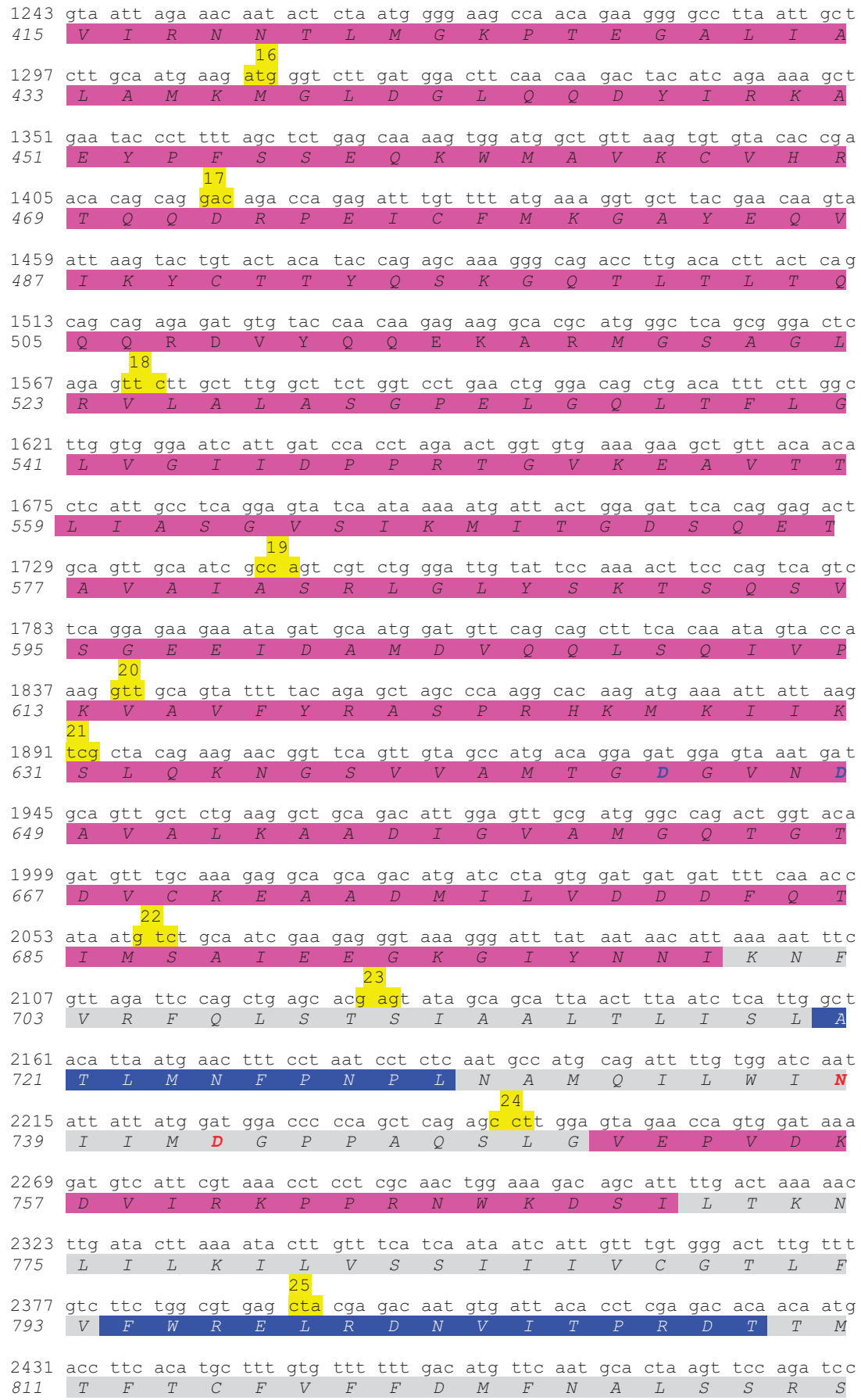

Figure 2 Continued

be addressed to the 3 ' end of the gene (namely the last 3 exons/ introns), where the alternative splicing generates the four different isoforms of SPCA1.

Few nonsense mutations were frequently reported in different families worldwide (reports with at least three cases): $115 C>T$ causing $\mathrm{R} 39 \mathrm{X} ;{ }^{6,18,19} 457 C>T$ causing $\mathrm{R} 153 \mathrm{X} ;{ }^{7,20-25} 1402 C>T$ causing R468X; ${ }^{7,26-28} 1516 C>T$ causing Q506X; ${ }^{29,30}$ and $2395 C>T$ causing $R 799 X .^{18,25,31-34}$ The above single residues represent 'hotspots' for mutations, and notably, they all occur at CpG sites where the possibility of mutations is higher. ${ }^{35}$ The fact that the majority of the mutations cause PTC through frameshift or single-base-pair substitution points to haploinsufficiency as the prevalent mechanism for the dominant inheritance of HHD. ${ }^{6,7}$ However, the possibility that at least some mutations cause the disease through a dominant negative mechanism cannot be excluded. $^{13}$

Interestingly, only one mutation was found in the most 3 ' region (exons 27-28) of the gene where differential splicing generates the four different transcripts. ${ }^{22}$ The single reported 


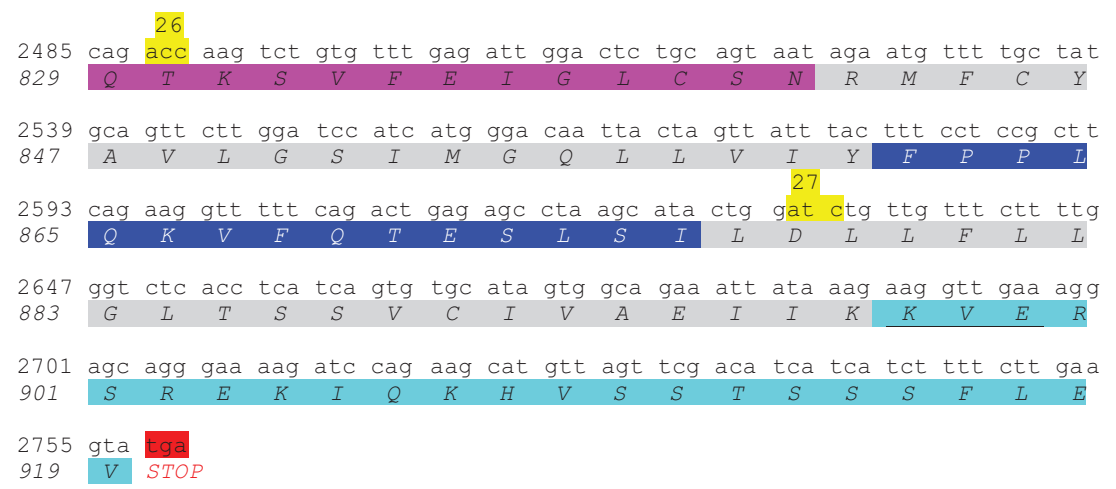

Figure 2 Continued

mutation, located in exon 27, was a nonsense mutation $(2660 C>A)$, which results in a stop codon (S887X) on the last transmembrane domain (M10). The mutation was found in a patient with a family history of HHD and suffering from classical HHD symptoms. ${ }^{22}$ Notably, this mutation caused the loss of the $\mathrm{C}$ termini of the SPCA1a, SPCA1b and SPCA1d isoforms but not of SPCA1c that does not include exon 27. Thus, SPCA1c, the shortest of the isoforms, misses the M10, and therefore, its stability and consequent ability to function as a $\mathrm{Ca}^{2+}$ pump is rather questionable. ${ }^{36}$ Nevertheless, if SPCA1c was effectively expressed, we can conclude that it is not functional enough to overcome the loss of the other three isoforms at least in the skin of the patient presenting the mentioned S887X mutation. ${ }^{22}$ Remarkably, this observation suggests that the mRNA encoding for SPCA1c is probably not translated as previously suggested. ${ }^{37}$

Furthermore, the mutation on exon 27 causes a reduction in SPCA1a, SPCA 1b and SPCA1d of 33, 52 and 63 amino acids, respectively, resulting in a partial truncation of the M10 and of the full cytoplasmic tail, similarly to what happens in SPCA1c (888 amino acids). Of consequence, this mutation is predicted to determine an incomplete expression and degradation of these SPCA1 isoforms thus resembling SPCA1c. Hereafter, we will no more consider SPCA1c in this discussion.

Splice variants differ in their C-terminal tail sequence (Figure 1c) and this is likely to be important for the functionality of the pump. Indeed, the C-terminal tail could have a role in mediating interactions with cytoplasmic effectors for intracellular signaling or for targeting the single isoforms to specific sub-organellar localization. Presently, no cytoplasmic interacting proteins have been found for the SPCA1 C-terminal domain. However, specific interactions of different SPCA1 tails with proteins involved in membrane trafficking (i.e., golgi matrix, $\mathrm{Ca}^{2+}$-binding proteins, Arf/Rab family members, cytoskeleton, etc.) could support a specific role for $\mathrm{C}$-terminal tail in the differential distribution of the protein at suborganellar level. This would in turn make them unique not only in triggering the $\mathrm{Ca}^{2+}$ influx into the golgi apparatus, but also in mediating different cytoplasmic signaling, thus orchestrating membrane trafficking at different levels along the secretory pathway. Obviously, these speculations need to be experimentally proven.

No further mutations have been discovered in the $3^{\prime}$-end of the ATP2C1 gene. In other words, once the ATP2C1 gene is mutated (independently if missense, nonsense, insertion and/or duplication or part of the primary sequence) the functionality of all the isoforms is equally compromised in HHD patients, and at least in the skin, levels of SPCA1 appear to be not sufficient. However, up-regulation of SERCA2 (the ER ATPase $\mathrm{Ca}^{2+}$ pump type 2) has been recently shown to (at least partly) compensate for the decreased levels of SPCA1 in HHD. ${ }^{38}$

Therefore, although SPCA1 is ubiquitously expressed, is there differential expression of the isoforms? Is the compensatory role for non-functional or depleted SPCA1 by other pumps tissue specific? Is there a general mechanism? If so, how does it work? Even if some tentative explanations have been given, ${ }^{38,39}$ these open questions need answers to fully understand the role and distribution of the SPCA1 isoforms.

\section{Missense Mutations on the ATP2C1 Gene}

Missense mutations generate single amino acid substitution, not leading to a PTC. A quick view on where the missense mutations occur along the ATP2C1 gene sequence gives an idea of their preferential distribution in few exons. In particular, almost two-thirds (63\%) of the missense mutations (31/49) localize in only five exons (exon 12, 13, 18, 21, and 23; Table 1). These exons encode for M4, P, ATP and M5/M6 domains, respectively (Figure 3). Amino acids located on M4, M5 and M6 (together with M8) transmembrane domains are critical for $\mathrm{Ca}^{2+} / \mathrm{Mn}^{2+}$ binding. It is not surprising that affecting residues in these regions, either directly involved in $\mathrm{Ca}^{2+}$-binding or compromising the stability or structure of the protein, may cause a severe effect on SPCA1 functionality, also without a reduction in its levels. Looking at the conservation across species we found high level of homology of these exons and conservativeness of the resulting amino acid coded by the codons where missense mutations occur (Figure 3), confirming their critical role.

Several residues have been reported to have a critical and direct role in $\mathrm{Ca}^{2+} / \mathrm{Mn}^{2+}$ binding, which are conserved throughout species ${ }^{40}$ and also in SPCA2, the homolog of SPCA $1{ }^{41}$ Missense mutation D778A in the yeast homolog (PMR1) resulted in a loss of function mutant apparently defective for the transport of both $\mathrm{Ca}^{2+}$ and $\mathrm{Mn}^{2+}$, whereas mutant Q783A displayed a differential sensitivity consistent with the selective loss of $\mathrm{Mn}^{2+}$ transport. ${ }^{42}$ In a similar study, 
Table 1 Mutations on the ATP2C1 gene reported in the literature

\begin{tabular}{|c|c|c|c|c|c|c|c|}
\hline Exon/Intron & Nucleotide change & Mutation & Number $^{a}$ & Codon $^{b}$ & Effect & Domain $^{\mathrm{c}}$ & References \\
\hline Exon 2 & 28delG/ins24bp ${ }^{d}$ & Deletion/insertion & 1 & & PTC & $\mathrm{N}$-ter & $6,23 \mathrm{~d}$ \\
\hline Exon $2^{d}$ & $115 \mathrm{C}>\mathrm{T}$ & Nonsense & 4 & R39X & PTC & $\mathrm{N}$-ter/s1 & $6,18,19$ \\
\hline Intron 2 & $117+2 T>G$ & Donor splice & 1 & & PTC(?) & $\mathrm{N}$-ter/s1 & 95 \\
\hline Intron 2 & $118-2 A>G$ & Acceptor splice & 1 & & PTC & $\mathrm{N}$-ter/s1 & 96 \\
\hline Intron 2 & $118-1 \mathrm{G}>\mathrm{A}$ & Acceptor splice & 3 & & & $\mathrm{~N}$-ter/s1 & $22 \mathrm{e}, 23,97 \mathrm{~d}, \mathrm{f}$ \\
\hline Exon $3^{d}$ & 134delG & Deletion & 1 & $45 \mathrm{Gfs} X 1$ & PTC & $\mathrm{N}$-ter/s1 & 98 \\
\hline Exon 3 & $163 C>T$ & Nonsense & 3 & R55X & PTC & $\mathrm{N}$-ter/s1 & $31,97 \mathrm{~d}, \mathrm{f}, 99$ \\
\hline Exon 3 & 168delC & Deletion & 1 & $?$ & PTC & $\mathrm{N}$-ter/s1 & 31 \\
\hline Exon 3 & $180 G>A^{d}$ & Nonsense & 1 & W60X & PTC & $\mathrm{N}$-ter/s1 & 97 \\
\hline Exon 3 & 185delAGTT & Deletion & 1 & $62 \mathrm{Kfs} \times 34$ & PTC & $\mathrm{N}$-ter/s1 & 28 \\
\hline Exon 3 & 212delT & Deletion & 1 & 71LfsX26 & PTC & M1 & $100 \mathrm{~g}$ \\
\hline Intron 3 & $235-2 A>G$ & Acceptor splice & 2 & & & M1 & 101,102 \\
\hline Exon 5 & 335delT & Deletion & 1 & 111LfsX19 & PTC & M2 & 23 \\
\hline Intron 5 & $360+1 G>A$ & Donor splice & 1 & & & M2 & 33 \\
\hline Intron 5 & $360+1 G>C$ & Donor splice & 1 & & Skip exon 5 & M2 & 24 \\
\hline Intron 5 & $360+2 T>A$ & Donor splice & 2 & & Skip exon 5 & M2 & 103 \\
\hline Intron 5 & $361-1 G>A^{d}$ & Acceptor splice & 1 & & PTC/skip exon 6 & M2 & 19 \\
\hline Intron $5^{\mathrm{d}}$ & $361-2 A>G^{d}$ & Acceptor splice & 1 & & PTC/loss exon 6 & $\mathrm{M} 2$ & 104 \\
\hline Exon 6 & $366 \mathrm{~T}>\mathrm{A}$ & Nonsense & 1 & Y122X & РТС & M2 & 105 \\
\hline Exon 7 & $457 \mathrm{C}>\mathrm{T}$ & Nonsense & 8 & R153X & PTC & A & $7,20,21,23-25$ e,106 \\
\hline Exon 7 & 490delT & Deletion & 1 & 163LfsX24 & PTC & A & 13 \\
\hline Exon 7 & 519insA & Insertion & 2 & 173LfsX3 & PTC & A & 13,33 \\
\hline Exon 7 & 520delC & Deletion & 1 & 174RfsX14 & РTC & $A$ & 24 \\
\hline Intron 7 & $531+2 T>A^{d}$ & Donor splice & 1 & & & A & 18 \\
\hline Exon 8 & $602 \mathrm{C}>\mathrm{T}^{\mathrm{h}}$ & Missense & 2 & $\mathrm{P} 201 \mathrm{~L}^{\mathrm{h}}$ & & $A$ & $6 \mathrm{~d}, 13$ \\
\hline Exon $8^{d}$ & $635 \mathrm{C}>\mathrm{A}$ & Nonsense & 1 & $\mathrm{~S} 212 \mathrm{X}$ & PTC & A & 96 \\
\hline Exon 8 & $661 A>C^{d}$ & Missense & 1 & T221P & $r 10$ & $A$ & 87 \\
\hline Exon 8 & 681dupA & Insertion & 1 & $227 \mathrm{Kfs} \times 13$ & PTC & $A$ & 24 \\
\hline Intron 8 & $688-1 G>A$ & Acceptor splice & 1 & & & $A$ & 13 \\
\hline Exon 9 & $689 G>A$ & Missense & 1 & G230D & & $A$ & 107 \\
\hline Exon 9 & 705 delA & Deletion & 1 & $235 T f s X 12$ & PTC & $A$ & 108 \\
\hline Exon 9 & $745 \mathrm{C}>\mathrm{T}$ & Nonsense & 1 & Q249X & PTC & S3 & 13 \\
\hline Exon 10 & 767insCCCT & Insertion & 1 & 256TfsX42 & PTC & S3 & 7 \\
\hline Exon 10 & $775 \mathrm{C}>\mathrm{T}$ & Nonsense & 1 & Q259X & PTC & S3 & 105 \\
\hline Exon 10 & $806 \mathrm{~T}>\mathrm{G}$ & Missense & 1 & L269R & & M3 & 105 \\
\hline Exon 10 & $832 G>A^{i}$ & $\begin{array}{l}\text { Missense/ } \\
\text { insertion }\end{array}$ & 2 & 278GfsX22 & & M3 & 91 \\
\hline Intron 10 & $832+3 A>T$ & Donor splice & 2 & & & M3 & 13 \\
\hline Intron 10 & $832+2 T>C$ & Donor splice & 1 & & Skip exon 10 & M3 & 20 \\
\hline Intron $10^{d}$ & $833-1 G>A^{d}$ & Acceptor splice & 1 & & & M3 & 6 \\
\hline Exon 11 & 836insT & Insertion & 1 & 279lfsX19 & PTC & M3 & 7 \\
\hline Exon 11 & $854 G>A$ & Nonsense & 1 & W285X & PTC & 12 & 95 \\
\hline Intron 11 & $899+1 G>T$ & Donor splice & 1 & & PTC & M4 & 24 \\
\hline Intron 11 & $899+1 G>C$ & Donor splice & 2 & & PTC & M4 & 109 \\
\hline Exon 12 & $910 \mathrm{G}>\mathrm{T}$ & Missense & 2 & A304S & & M4 & $7 \mathrm{j}, 20$ \\
\hline Exon 12 & $920 \mathrm{C}>\mathrm{T}$ & Missense & 1 & P307L & & M4 & 104 \\
\hline Exon 12 & $920 \mathrm{C}>\mathrm{A}$ & Missense & 1 & P307H & & M4 & 36 \\
\hline Exon 12 & 923delAAG & Deletion & 1 & 308 delE $^{\mathrm{k}}$ & & M4 & 28 \\
\hline Exon 12 & $925 \mathrm{G}>\mathrm{T}$ & Missense & 1 & G309C & & M4 & 13 \\
\hline Exon 12 & 932 del21bp ${ }^{d}$ & Deletion & 1 & $311 \mathrm{del}^{\mathrm{d}}$ & & $\begin{array}{l}\text { IV14 } \\
\text { M4 }\end{array}$ & 18 \\
\hline Exon 12 & $935 \mathrm{~T}>\mathrm{C}$ & Missense & 1 & I312T & & M4 & 110 \\
\hline Exon 12 & 950del9bp/ins $24 b p^{\prime}$ & Deletion/insertion & 1 & 318-320del/ins' & & S4 & 20 \\
\hline Exon 12 & $953 \mathrm{~T}>\mathrm{C}$ & Missense & 1 & L318P & & S4 & 7 \\
\hline Exon 12 & 956delC & Deletion & 1 & 319AfsX3 & PTC & S4 & 24 \\
\hline Exon 12 & 1001 delA & Deletion & 1 & $333 K f s X 12$ & PTC & S4 & 13 \\
\hline Exon 12 & $1004 \mathrm{~T}>\mathrm{C}$ & Missense & 1 & L335P & & S4 & 111 \\
\hline Exon 12 & $1022 T>C$ & Missense & 1 & L341P & & S4 & 13 \\
\hline Intron 12 & $1024+1 G>A^{d}$ & Donor splice & 1 & & PTC/skip exon 12 & $\begin{array}{l}54 \\
\text { S4 }\end{array}$ & 21 \\
\hline Exon 13 & $1031 G>A^{d, h}$ & Missense & 1 & C344Y $Y^{d, h}$ & rTU/SKIP exUा IL & $\begin{array}{c}34 \\
P\end{array}$ & 6 \\
\hline Exon 13 & $1042 \mathrm{~T}>\mathrm{C}$ & Missense & 1 & C348R & & $P$ & 105 \\
\hline Exon 13 & 1045delT & Deletion & 1 & $348 \mathrm{Cfs} \times 6$ & PTC & $\mathrm{P}$ & 13 \\
\hline Exon 13 & $1049 A>T$ & Missense & 3 & D350V & & $\mathrm{P}$ & 28 \\
\hline Exon 13 & $1055 C>T^{d}$ & Missense & 1 & T352l & & $\mathrm{P}$ & 112 \\
\hline Exon 13 & $1058 \mathrm{G}>\mathrm{T}^{\mathrm{d}}$ & Missense & 1 & G353V & & P & 30 \\
\hline Exon $13^{d}$ & 1067delC & Deletion & 1 & $356 \mathrm{Tfs} \times 3$ & PTC & $P$ & 96 \\
\hline Exon 13 & 1068del16bp ${ }^{m}$ & Deletion & 1 & $356 \mathrm{Tfs} \times 60$ & PTC & $P$ & 113 \\
\hline Exon 13 & 1085insA & Insertion & 1 & $363 \mathrm{Tf} \times 11$ & PTC & $\mathrm{P}$ & 29 \\
\hline Exon 13 & $1087 A>G$ & Missense & 1 & T363A & & $P$ & 114 \\
\hline Exon 13 & 1089delTCAC & Deletion & 4 & $363 T f s \times 21$ & PTC & $\mathrm{P}$ & $13,23,28,115$ \\
\hline Exon 14 & $1218 G>C^{d}$ & Missense & 1 & F406D & Skin exon 14 & $P$ & 19 \\
\hline Exon 15 & $1231 T>0$ & Missense & 1 & C411R & SkIp exon 14 & 8 & 13 \\
\hline Exon $15^{\mathrm{d}}$ & $1250 G>A^{d}$ & Missense & 3 & R417K & & P & 32 \\
\hline Intron 15 & $1308+1 \mathrm{G}>\mathrm{A}$ & Donor splice & 1 & & & P & 36 \\
\hline Intron 15 & $1309-1 G>A$ & Acceptor splice & 1 & & & P & 13 \\
\hline Intron 15 & $1309-4 a>t / 1309-2 a>q$ & Acceptor splice & 1 & & Skip exon 16 & P & 7 \\
\hline Exon 16 & $1327 \mathrm{C}>\mathrm{T}^{\mathrm{d}}$ & Nonsense & 1 & $Q 443 X^{d}$ & PTC & $P$ & 6 \\
\hline Exon 16 & $1388 T>G$ & Missense & 1 & V463G & & $P$ & 20 \\
\hline
\end{tabular}


Table 1 (Continued)

\begin{tabular}{|c|c|c|c|c|c|c|c|}
\hline Exon/Intron & Nucleotide change & Mutation & Number $^{a}$ & Codon $^{b}$ & Effect & Domain $^{c}$ & References \\
\hline Exon 16 & $1402 C>T$ & Nonsense & 4 & $\mathrm{R} 468 \mathrm{X}$ & PTC & $\mathrm{P}$ & $7,26-28$ \\
\hline Exon 16 & $1413 G>C$ & Missense & 1 & Q471H & & $?$ & 110 \\
\hline Intron 16 & $1415-2 A>C$ & Acceptor splice & 1 & & PTC/skip exon 17 & $?$ & 104 \\
\hline Exon 17 & 1413del28bp ${ }^{n}$ & Deletion & 2 & 472DfsX14 & PTC & ? & 116 \\
\hline Exon 17 & $1431 \mathrm{~T}>\mathrm{A}$ & Nonsense & 1 & C477X & PTC & $?$ & 117 \\
\hline Exon 17 & 1455 del $^{d}$ & Deletion & 1 & 485QfsX1 & PTC & N? & 118 \\
\hline Exon 17 & $1462 \mathrm{del}^{\mathrm{d}, \mathrm{o}}$ & Deletion & 1 & $488 \mathrm{del}^{\circ}$ & & $\mathrm{N}$ & 104 \\
\hline Exon 17 & $1469 G>T$ & Missense & 1 & C490F & & $\mathrm{N}$ & 31 \\
\hline Exon 17 & 1508delCTCA ${ }^{d}$ & Deletion & 1 & 503TfsX32 & PTC & $\mathrm{N}$ & 18 \\
\hline Exon 17 & $1510 \mathrm{C}>\mathrm{T}$ & Nonsense & 1 & Q504X & PTC & $\mathrm{N}$ & 36 \\
\hline Exon 17 & $1516 \mathrm{C}>\mathrm{T}$ & Nonsense & 4 & Q506X & PTC & $\mathrm{N}$ & $29,30 \mathrm{~d}$ \\
\hline Exon 17 & 1523delAT & Deletion & 2 & 508DfsX23 & PTC & $\mathrm{N}$ & 97,104 \\
\hline Exon 17 & 1566delCA & Deletion & 1 & 522LfsX9 & PTC & $\mathrm{N}$ & 7 \\
\hline Intron 17 & $1570+2 T>C$ & Donor splice & 1 & & PTC & $\mathrm{N}$ & 24 \\
\hline Exon 18 & $1582 G>C$ & Missense & 1 & A528P & & $\mathrm{N}$ & 23 \\
\hline Exon 18 & $1588 G>C$ & Missense & 1 & G530R & & $\mathrm{N}$ & 95 \\
\hline Exon 18 & $1685 C>G$ & Nonsense & 3 & S562X & PTC & $\mathrm{N}$ & $6 \mathrm{~d}, 13,28$ \\
\hline Exon 18 & $1709 \mathrm{C}>\mathrm{T}^{\mathrm{d}, \mathrm{h}}$ & Missense & 2 & $\mathrm{~T}^{2} 70 \mathrm{l}^{\mathrm{d}, \mathrm{h}}$ & & $\mathrm{N}$ & 6 \\
\hline Exon 18 & 1723delG & Deletion & 1 & 574QfsX24 & PTC & $\mathrm{N}$ & 13 \\
\hline Exon 18 & $1738 A>G$ & Missense & 2 & I580V & & $\mathrm{N}$ & 13,31 \\
\hline Intron 18 & $1694-1 G>A$ & Acceptor splice & 1 & & & $\mathrm{~N}$ & 6 \\
\hline Exon 19 & $1751 \mathrm{~T}>\mathrm{C}$ & Missense & 1 & L584P & & $\mathrm{N}$ & 21 \\
\hline Exon 19 & 1782delAGTC & Deletion & 1 & $593 S f s \times 5$ & PTC & $\mathrm{N}$ & 119 \\
\hline Exon 19 & $1816 \mathrm{C}>\mathrm{T}$ & Nonsense & 1 & Q606X & PTC & $\mathrm{N}$ & 13 \\
\hline Intron 19 & $1839+2$ ins $^{d}$ & Donor splice & 1 & & PTC & $\mathrm{N}$ & 23 \\
\hline Intorn 19 & $1840-1 G>C$ & Acceptor splice & 1 & & & $\mathrm{~N}$ & 98 \\
\hline Exon 20 & $1854 G>A^{d}$ & Missense & 1 & R619K & & $\mathrm{N}$ & 87 \\
\hline Exon 20 & 1869delG & Deletion & 1 & 623RfsX2 & PTC & $\mathrm{N}$ & 33 \\
\hline Exon 20 & 1874delA & Deletion & 1 & M626X & PTC & $\mathrm{N}$ & 120 \\
\hline Exon 20 & 1875delG & Deletion & 1 & M626X & PTC & s5 & 7 \\
\hline Intron 20 & 1890+1delGTGAG/ins & Donor splice & 1 & & & s5 & $22 \mathrm{e}$ \\
\hline Intron 20 & $1891-1 \mathrm{G}>\mathrm{T}$ & Acceptor splice & 1 & & & s5 & 121 \\
\hline Exon 21 & $1897 \mathrm{C}>\mathrm{T}$ & Nonsense & 1 & Q633X & PTC & s5 & 122 \\
\hline Exon 21 & 1914del/ins ${ }^{d}$ & Deletion/insertion & 1 & $638 \mathrm{Vfs} 10 \mathrm{X}$ & PTC & s5 & $123 \mathrm{~d}$ \\
\hline Exon 21 & $1922 T>G$ & Missense & 1 & M641R & & s5 & 7 \\
\hline Exon $21^{d}$ & $1931 A>G$ & Missense & 1 & D644G & & s5 & 33 \\
\hline Exon 21 & $1933 G>A$ & Missense & 1 & G645R & & s5 & 7 \\
\hline Exon 21 & $1934 G>T^{d}$ & Missense & 1 & G645V & & s5 & 124 \\
\hline Exon 21 & $1942 G>T$ & Missense & 1 & D648Y & & s5 & 104 \\
\hline Exon 21 & $1952 C>A$ & Missense & 1 & A651D & & s5 & 105 \\
\hline Exon 21 & $1982 \mathrm{~T}>\mathrm{G}$ & Missense & 1 & M661R & & s5 & 113 \\
\hline Exon 21 & 1983delG & Deletion & 1 & $661 \mathrm{Mfs} X 14$ & PTC & s5 & 7 \\
\hline Exon 21 & 2023delA ${ }^{d}$ & Deletion & 1 & $675 \mathrm{Mfs} X$ & PTC & s5 & 18 \\
\hline Exon 21 & 2025delG & Deletion & 1 & $675 \mathrm{Mfs} X 2$ & PTC & s5 & 105 \\
\hline Intron $21^{d}$ & $2058(-17 C>T)^{d}$ & Acceptor splice & 1 & & PTC & s5 & 97 \\
\hline Intron $21^{\mathrm{d}}$ & $2058-1 G>C^{d}$ & Acceptor splice & 1 & & & s5 & 28 \\
\hline Intron 21 & $2058-1 \mathrm{G}>\mathrm{A}$ & Acceptor splice & 1 & & Skip exon 22 & s5 & 7 \\
\hline Exon 22 & $2068 G>T$ & Nonsense & 1 & E690X & PTC & s5 & 110 \\
\hline Exon 22 & 2111 ins & Insertion & 1 & 704RfsX23 & PTC & M5 & 20 \\
\hline Exon 22 & $2126 \mathrm{C}>\mathrm{T}$ & Missense & 3 & T709M & & M5 & $7,28,107$ \\
\hline Intron 22 & $2127+1 G>A^{d}$ & Donor splice & 2 & & Skip exon 23 (?) & M5 & $28 \mathrm{~d}, 125 \mathrm{~d}$ \\
\hline Intron $22^{d}$ & $2126(+5 G>A)^{d}$ & Donor splice & 1 & & PTC & M5 & 97 \\
\hline Exon 23 & $2132 T>G$ & Missense & 1 & I711R & & M5 & 31 \\
\hline Exon 23 & $2141 \mathrm{~T}>\mathrm{A}$ & Nonsense & 1 & L714X & PTC & M5 & 23 \\
\hline Exon 23 & 2164insACAT & Insertion & 1 & $722 \mathrm{Lfs} \times 6$ & PTC & 13 & 122 \\
\hline Exon 23 & $2198 A>G$ & Missense & 1 & Q733R & & M6 & 31 \\
\hline Exon 23 & 2215delATT & Deletion & 1 & 739dell & & M6 & 20 \\
\hline Exon 23 & $2224 G>T$ & Missense & 1 & D742Y & & M6 & 13 \\
\hline Exon 23 & 2227 delG $^{d}$ & Deletion & 1 & $743 \mathrm{Gfs} \times 8$ & РТC & M6 & 6 \\
\hline Exon 23 & $2231 C>G$ & Missense & 2 & P744R & & M6 & $7 \mathrm{p}, 20$ \\
\hline Exon 23 & 2235insC & Insertion & 1 & 746AfsX10 & PTC & M6 & 107 \\
\hline Exon 23 & $2236 G>A^{d}$ & Missense & 1 & $A 746 T^{d}$ & & M6 & 18 \\
\hline Exon 23 & $2237 \mathrm{C}>\mathrm{T}^{\mathrm{d}}$ & Missense & 1 & A746V & & M6 & 34 \\
\hline Intron 23 & $2243+2 \mathrm{~T}>\mathrm{C}$ & Donor splice & 1 & & РТC & M6 & 104 \\
\hline Exon 24 & $2246 \mathrm{~T}>\mathrm{G}$ & Missense & 1 & L749R & & M6 & 20 \\
\hline Exon 24 & 2251delGT & Deletion & 1 & $751 \mathrm{Vfs} \times 5$ & PTC & s6 & 126 \\
\hline Exon 24 & 2264delA & Deletion & 2 & $755 \mathrm{Dfs} X 17$ & PTC & s6 & 25,33 \\
\hline Exon 24 & 2303delAC & Deletion & 2 & 768 DfsX4 & PTC & s6 & 7,20 \\
\hline Exon 24 & 2339delTTGT ${ }^{d}$ & Deletion & 1 & $780 \mathrm{Lfs} X 3$ & РTC & M7 & 19 \\
\hline Exon 24 & 2357delTT & Deletion & 1 & 786 Ifs X10 & РТC & M7 & 7 \\
\hline Exon 24 & $2365 G>A$ & Missense & 1 & G789R & & M7 & 13 \\
\hline Exon 24 & 2371delTTGT & Deletion & 4 & 791LfsX12 & PTC & M7 & $7,20,127 \mathrm{~d}$ \\
\hline Exon 24 & 2374delTTTG & Deletion & 10 & $792 \mathrm{Ffs} X 10$ & PTC & M7 & $6 \mathrm{~d}, 7,13,23,31,117,128,129 \mathrm{~d}, 130$ \\
\hline Exon 24 & 2375delTTGT & Deletion & 2 & $792 \mathrm{Ffs} 44$ & PTC & $\mathrm{M7}$ & \\
\hline Exon 24 & $2384 G>A$ & & 1 & W795X & PTC & 14 & $22 \mathrm{e}$ \\
\hline Exon 25 & $2395 \mathrm{C}>\mathrm{T}$ & $\begin{array}{l}\text { Nonsense } \\
\text { Nonsense }\end{array}$ & 14 & R799X & PTC & 14 & $16 \mathrm{~d}, 25,31-34 \mathrm{~d}$ \\
\hline Exon $25^{d}$ & $2412 \mathrm{delT}$ & Nonsense & 14 & $8031 \mathrm{fs} \times 7$ & PTC & $\begin{array}{l}14 \\
14\end{array}$ & 102 \\
\hline Exon 25 & $2416 \mathrm{C}>\mathrm{T}$ & Nonsense & 1 & R806X & PTC & $\begin{array}{l}14 \\
14\end{array}$ & 13 \\
\hline
\end{tabular}


Table 1 (Continued)

\begin{tabular}{|c|c|c|c|c|c|c|c|}
\hline Exon/Intron & Nucleotide change & Mutation & Number $^{a}$ & Codon $^{b}$ & Effect & Domain $^{c}$ & References \\
\hline Exon 25 & 2422delAC & Deletion & 2 & $808 T f s X 10$ & PTC & 14 & 33 \\
\hline Exon 25 & 2445del10bp & Deletion & 3 & 814CfsX7 & PTC & M8 & 131 \\
\hline Exon 25 & 2454delT & Deletion & 1 & $818 \mathrm{Ffs} X 6$ & PTC & M8 & 31 \\
\hline Exon 25 & 2454dupT & Insertion & 1 & D819X & PTC & M8 & 28 \\
\hline Exon 25 & 2460delG & Deletion & 1 & 820MfsX4 & PTC & M8 & 21 \\
\hline Exon 25 & $2468 A>C^{d}$ & Missense & 2 & A823E & & M8 & 97 \\
\hline Exon 26 & 2529delGT & Deletion & 1 & $843 \mathrm{Mfs} X 27$ & PTC & M9 & 7 \\
\hline Exon 26 & 2558del10bp ${ }^{q}$ & Deletion & 1 & 853MfsX17 & PTC & M9 & 31 \\
\hline Exon 26 & $2593 C>T^{d}$ & Nonsense & 2 & Q865X $X^{d}$ & PTC & 15 & 6,112 \\
\hline Exon $26^{d}$ & $2597 A>C$ & Missense & 1 & K866T & & 15 & 96 \\
\hline Intron 26 & 2630-1delG & Acceptor splice & 2 & & & M10 & $6 \mathrm{~d}, 13$ \\
\hline Exon 27 & $2660 \mathrm{C}>\mathrm{A}$ & Nonsense & 1 & S887X & PTC & M10 & $22 \mathrm{e}$ \\
\hline
\end{tabular}

Appendix 1

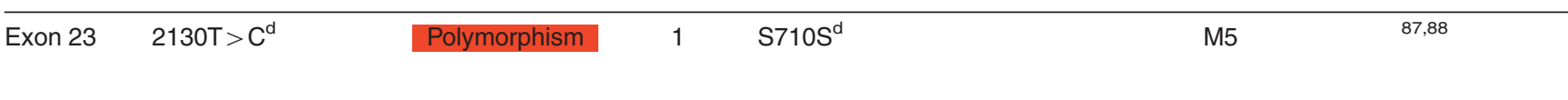

Appendix 2

\begin{tabular}{llrrr}
\hline Number & Mutation & $\%$ & PTC & \% \\
\hline 59 & Deletion/insertion & 35.54 & 55 & 59.78 \\
24 & Nonsense & 14.46 & 24 & 0 \\
49 & Missense & 29.52 & 13 & 0.00 \\
34 & Acceptor/donor splice & 20.48 & 92 & 14.13 \\
166 & & & 55.42
\end{tabular}

Appendix 3

\begin{tabular}{llllllllllllll}
\hline $\begin{array}{l}\text { Normal } \\
\text { p.309 }\end{array}$ & G & G & P & I & V & V & T & V & T & L & A & L & p.320 \\
c.925 & GGT & CTC & CCC & ATT & GTG & GTC & ACA & GTG & ACG & CTA & GCT & CTT & C.960 \\
c.925 & GGT & CTC & CTA & GCT & CTT & c.939 & & & & \\
p.309 & G & L & L & A & L & p.313 & & & \\
Mutant & & & & & & & &
\end{tabular}

Human ATP2C1 gene mutations were summarized. The mutations are grouped for deletion/insertion (azure), nonsense (orange), missense (yellow), acceptor/donor splice (green). Nucleotides are reported in italic along the table as well as in the figure legend and along the full manuscript. We found some reported mutations were inaccurate or not unified. Therefore, we revised or collated some descriptions according to the reported cDNA reference sequence (GenBank accession No. NM_AF181120). ${ }^{7}$

Appendix 1: A polymorphism was wrongly reported to be a new mutation $2323 C>T$ generating $Y 711 \mathrm{H} .{ }^{87}$ After careful check with the correct reading frame this was not a mutation but a polymorphism. ${ }^{88}$

Appendix 2: A resuming panel graphs the amount and relative percentage of each kind of mutations and PTC.

Appendix 3: The 884-904delCCATTGTGGTCACAGTGACGC mutation and consequent amino acid $296-302$ dellVVTVTL was incorrectly reported, ${ }^{18}$ while it referred to as a $21 \mathrm{bp}$ deletion located at $932-952$ and amino acid $311-317$ delPIVVTVT. This mutation did not generate the reported missense mutation P295V; ${ }^{18}$ the first ' $C$ ' of the codon 311 (encoding for a proline, $\mathrm{P}$ ) recombined with 'TA' of codon 318 (leucine, L) generating the codon CTA which encoded for a leucine.

${ }^{a}$ Number of reported cases of patients presenting the mutation.

${ }^{\mathrm{b}}$ Missense mutations causing an amino acid substitution in extremely conserved residue through all the ATPases and in different species are highlighted in light pink. ${ }^{23}$

${ }^{c}$ Putative protein domain prediction is based on the position of the equivalent residue within the structure of ATP2A1 (SERCA1).

dUsing the running correct coding sequence and relative reading frame of the ATP2C1 gene (Ref. NG_007379.1) we unified the position of the mutation site, protein change, exon/intron location all over the reported mutations. In doing this we found few mutations published as new which were already known.

eThe same authors published their findings in two identical papers on different journals. ${ }^{22,89}$

${ }^{f}$ Due to incorrect interpretation of discovered mutations the authors reported as new previously reported mutations. ${ }^{90}$

gIn their manuscript, the authors reported a previously described mutation. A mistake on referring to this mutation was recently reported (see erratum in Acta Derm Venereol 2015; 95: 1040).

${ }^{\mathrm{h}} \mathrm{P} 201 \mathrm{~L}, \mathrm{C} 344 \mathrm{Y}$, and T570I, respectively, represent mutations P185L, C328Y, and T554I, originally reported by Sudbrak et al. ${ }^{6}$ Mutation nomenclature has now been updated with respect to the $5^{\prime}$-end sequence published by Hu et al ${ }^{7}$ and the results of $5^{\prime}$ RACE-PCR experiments from Fairclough et al. ${ }^{36}$

iThe missense mutation $832 G>A$ causing the nucleotide change G278R generated an aberrant splicing with a resulting insertion of the first 11 bp (GTAAGAGAAGA) from intron 10 between the mutated exon10 and the exon 11 (see Figure 5 in Chao et al) ${ }^{91}$ causing a PTC.

'This mutation was incorrectly reported as A304T by Hu et al ${ }^{7}$ as previously reported. ${ }^{20}$

${ }^{\mathrm{k}} \mathrm{E} 308$, and not G309 (which are both $\mathrm{Ca} 2+-$ binding site residue), was deleted.

'950delCGCTAGCTCTT>CT > insCCACAATGTGTTGGTGTTATGAGAAT (underlined are the deleted/inserted nucleotides) generates the in frame 318delLAL/ insTMCWCYEN

m1068deIAAGAATGAAATGACTG.

"nTe delGACAGACCAGAGATTTGTTTTATGAAAG cause a frame shift and PTC.

In frame delAAGTACTGTACTACATACCAGAGC with amino acid delKYCTTYQS.

${ }^{\mathrm{P}}$ This mutation was incorrectly reported as $\mathrm{P} 724 \mathrm{R}$ by Hu et $\mathrm{al}^{\mathrm{P}}$ as previously reported. ${ }^{20}$

${ }^{\mathrm{q}}$ The deleted sequence is TGGGACAATT. 
Canis lupus LAVAA IPEGLP IVVTVTLALGVMRMVKKRAIVKKLPIVETL

Mus musculus LAVAA IPEGLP IVVTVTLALGVMRMVKKRA IVKKLPIVETL Rattus norvegicus LAVAA IPEGLP IVVTVTLALGVMRMVKKRA IVKKLP IVETL

Danio rerio LAVAAIPEGLPIVVTVTLALGVMRMVKKRAIVKKLPIVETL

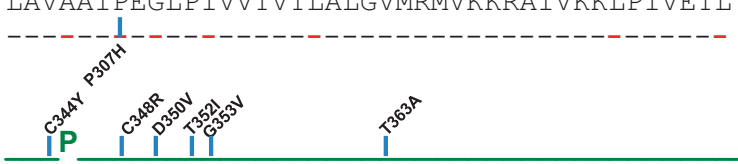

Homo sapiens GCCNVICSDKTGTLTKNEMTVTH IFT SDGLHAEVTGVGYNQFGEVIVDGDVVHGFYNPAV Pan troglodites GCCNVICSDKTGTLTKNEMTVTH IFT SDGLHAEVTGVGYNQFGEVIVDGDVVHGFYNPAV

Canis lupus GCCNVICSDKTGTLTKNEMTVTHIFTSDGLHAEVTGVGYNPFGEVIVDGDVVHGFYNPAV Mus musculus GCCNVICSDKTGTLTKNEMTVTHILTSDGLHAEVTGVGYNQFGEVIVDGDVVHGFYNPAV Rattus norvegicus GCCNVICSDKTGTLTKNEMTVTHILTSDGLHAEVTGVGYNQFGEVIVDGDVVHGFYNPAV

Danio rerio SCCNVICSDKTGTLTKNEMTVTHLFTSDGQHAEVTGIGYNNSGE I LMDGEVVHGISNTS I

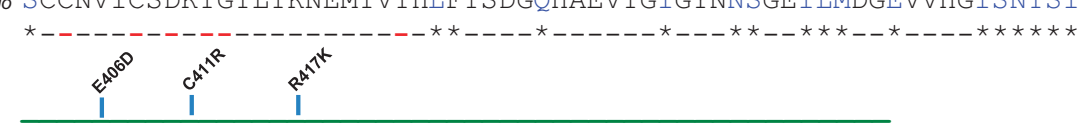

Homo sapiens SRIVEAGCVCNDAVIRNNTLMGKPTEGALIALAMKMGLDGLQQDYIRKA

Pan troglodites SRIVEAGCVCNDAV IRNNTLMGKPTEGAL IALAMKMGLDGLQQDY IRKA

Canis lupus SRIVEAGCVCNDAV I RNNTLMGKPTEGAL IALAMKMGLDGLQQDYVRKA

Mus musculus SRIVEAGCVCNDAVIRNNTLMGKPTEGALIALAMKMGLDGLQQDY IRKA

Rattus norvegicus SRIVEAGCVCNDAVIRNNTLMGKPTEGAL IALAMKMGLDGLQQDYIRKA

Danio rerio SKIVEVGCVCNDAMIRHNTLMGRPTEGALIALAMKMGLEGLQHEFVRLE

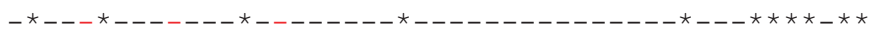

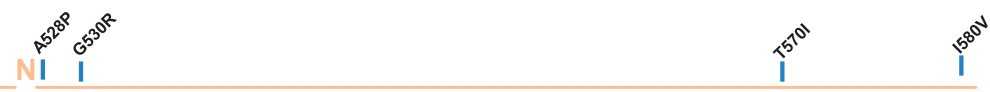

Homo sapiens VLALASGPELGQLTFLGLVGI IDPPRTGVKEAVTTLIASGVSIKMITGDSQETAVAI Pan troglodites VLALASGPELGQLTFLGLVGI IDPPRTGVKEAVTTLIASGVS IKMITGDSQETAVAI

Canis lupus VLALASGPELGQLTFLGLVGI I DPPRTGVKEAVTTLIASGVS I KMITGDSQETAVA I Mus musculus VLALASGPELGQLTFLGLVGI IDPPRTGVKEAVTTLIASGVSIKMITGDSQETAIA I Rattus norvegicus VLALASGPDLGQLTFLGLVGI IDPPRTGVKEAVTTLIASGVS IKMITGDSQETAIA I

Danio rerio VLALASGSEMGS LTFLGLVGI IDP PRAGVKEAVATLISSGVAVKMVTGDSEETAVAI $-------* \star \star-\star--------------*------*---\star---\star \star---\star----*---\star--$

S5

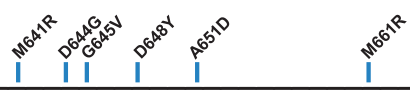

Homo sapiens SLQKNGSVVAMTGDGVNDAVALKAADIGVAMGQTGTDVCKEAADMILVDDDFQT IM

Pan troglodites SLQKNGSVVAMTGDGVNDAVALKAADIGVAMGQTGTDVCKEAADMI LVDDDFQT IM

Canis lupus SLQKNGSVVAMTGDGVNDAVALKAAD I GVAMGQTGTDVCKEAADMILVDDDFQT IM

Mus musculus SLQKNGAVVAMTGDGVNDAVALKAADIGVAMGQTGTDVCKEAADMILVDDDFQT IM Rattus norvegicus SLQKNGSVVAMTGDGVNDAVALKAADI GVAMGQTGTDVCKEAADMILVDDDFQT IM Danio rerio SLQNIGAVVAMTGDGVNDAVALKAADIGVAMGQTGTDVCKEAADMI LVDDDFQT IL
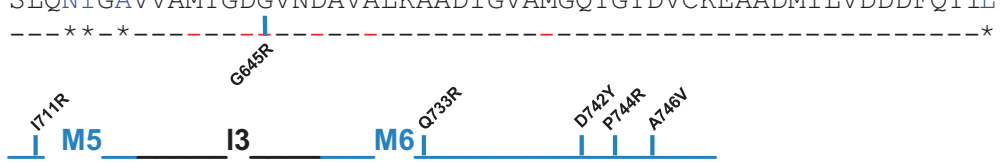

Homo sapiens SIAALTLISLATLMNEPNPLNAMQILWINI IMDGPPAQSL Pan troglodites S IAALTL I SLATLMNF PNP LNAMQI LWINI IMDGPPAQSL

Canis lupus SIAALTLISLATLMNFPNPLNAMQI LWIN I IMDGPPAQSL Mus musculus S IAALTL I SLATLMNFPNPLNAMQI LWINI IMDGPPAQSL Pan troglodites S IAALTL IS LATLMNFPNPLNAMQ I LWIN I IMDGPPAQSL

Danio rerio SIAALTL ISLATLLNF PNPLNAMQ ILW INI IMDGPPAQSL

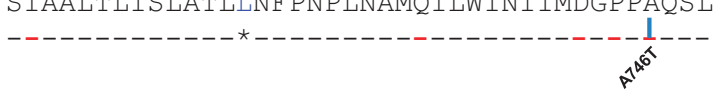

Figure 3 Missense mutations mostly affect critical SPCA1 domains. Most of the missense mutations localize on exons 12, 13-16, 18, 21, and 23 of ATP2C1 gene coding for the indicated protein domains (M4, P, N, S5, M5-M6) crucial for the protein enzymatic activity and $\mathrm{Ca}^{2+} / \mathrm{Mn}^{2+}$ binding. In human HHD patients the missense mutations cause the indicated amino acid changes, supporting a crucial role for them in functionality of the SPCA1. Of note, the mutations localize on highly conserved residue/codon between the considered species, highlighting the importance of those encoded amino acids. In blue are indicated the different amino acids compared to the human sequence, in red where the mutations occur. Alignments were obtained from Ensembl database as described in Figure 1 
G783 and V335 in PMR1 showed conformation-sensitive packing at the cytoplasmic interface, suggesting that these residues are important in organizing the entry gate for $\mathrm{Mn}^{2+}$ ions. ${ }^{43}$ Missense mutation can also cause a change in protein conformation affecting possible interaction of SPCA1 with other proteins, like cofilin, which has been found to be crucial in recruiting actin to mediate the cargo sorting that interact directly with SPCA1 nucleotide domain through an interaction mediated by $\mathrm{Ca}^{2+}$ ions. ${ }^{44}$

Remarkably, these examples highlight how a possible missense mutation can affect SPCA1 resulting not only in a less efficient pump, but also accounting for its diminished ability to interact with other proteins. In some HHD patients this scenario results in a severe etiology, comparable to the situation where mutations on ATP2C1 gene cause PTC, but in the presence of unaffected levels of total protein expression.

\section{ATP2C1 Gene: Human Versus Murine}

Another aspect to highlight is the different etiology occurring between humans and mice as a consequence of ATP2C1 mutations. In humans, HHD rarely degenerates in skin cancer although squamous cell carcinoma and basal cell carcinoma arising in lesions of HHD have been described in the literature. ${ }^{45-47}$ However, which is the relationship, if any, between skin cancer development and HHD has not been deeply investigated. To our knowledge, there are no reports of melanoma or non-cutaneous malignant neoplasms associated with HHD.

On the other side, heterozygotic mice exhibit no evidence of skin lesions indicative of HHD, meanwhile generated skin cancer in adult. ${ }^{48,49}$ Null mutant embryos exhibited growth retardation, failure of neural tube closure, but normal hematopoiesis and cardiovascular development, resulting in embryonic lethality. ${ }^{48}$ At subcellular level, golgi membranes of Spca1 ${ }^{-1-}$ embryos were dilated, had fewer stacked leaflets, and were expanded in amount, consistent with increased golgi biogenesis. ${ }^{48}$ Increased golgi-associated vesicles and a marked reduction of ribosomes on rough ER were also observed. ${ }^{48}$ Coated pits, junctional complexes, desmosomes, and basement membranes appeared normal in mutant embryos, indicating that processing and trafficking of proteins in the secretory pathway was not massively impaired. ${ }^{48}$ However, apoptosis was increased, possibly the result of secretory pathway stress, and a large increase in cytoplasmic lipid was observed in mutant embryos, consistent with impaired handling of lipids by the golgi. ${ }^{48}$ Possibly, reduction of the proper levels of Spca1 resulted in alteration in the activity of the glycosyl-transferases due to a decreased accumulation of $\mathrm{Mn}^{2+}$ in the golgi lumen, which could contribute to the secretory pathway stress as well as apoptosis due to a decreased of $\mathrm{Ca}^{2+}$ handling.

Recently, an important difference between human and murine genomes that could explain the differential outcome of mutations in the two species has been reported. ${ }^{50}$ The Atp2c1 gene in mice expresses only one isoform of the gene, not presenting alternative splicing events at the $3^{\prime}$-end of the gene like the human counterpart. The last exon of the ATP2C1 human gene overlapped in the antisense direction with the last exon of the ASTE1 (also named HTO01) gene, which codes for a protein of unknown function. ${ }^{50}$ ATP2C1/ASTE1 overlap (not present in the murine genome) could have a role in regulating isoform expression, or more largely, the full SPCA1 expression. ${ }^{50}$ This sort of regulation could have in turn a protective role that is imbalanced once one of the two alleles is mutated in humans, whereas not in mice. ${ }^{50}$

\section{Putative Interacting Domains in the C-terminal Cytosolic Tails of SPCA1 Isoforms}

As suggested above, different SPCA1 isoforms due to their unique $\mathrm{C}$ termini could have a different pattern of distribution and functional roles. The primary sequence of the C-terminal tail of each isoform is reported in Figure 1c. An intriguing possibility is that the uniqueness of their function is a consequence of the interaction with different protein partners. It is well known that many resident proteins display in their tails, motifs like sorting signals responsible for retrieval or retention. For instance, the C-terminal KKXX motif, identified in many ER-localized type I membrane proteins, can function as an ER-retention signal to retrieve ER-resident membrane proteins from the golgi via a direct interaction with coat protein complex I (COPI) coatomer. ${ }^{51-53}$ Another example is the semiconserved (F/L)-(L/V)-(S/T) motif, which has been shown to function as a golgi-retention signal by interacting with COPI vesicles via Vps74p and thereby maintaining the steady golgi localization of glycosyl-transferases in yeast. ${ }^{54}$ Recently, a conserved retention function and COPI-binding ability for the $\mathrm{KXD} / \mathrm{E}$ motif has been shown in the golgi apparatus through the evolution in different species and in many endomembrane proteins. ${ }^{55,56}$

Using bioinformatics tools (see Figure 4 legend), we analyzed the SPCA1 sequences in the areas corresponding to the terminal exons 26-28 in terms of secondary structure propensity (Figure 4a), predicted transmembrane helices topology (Figure 4b), and interaction with putative protein partners (Figure $4 \mathrm{c}$ ). We found that the KXD/E motif is present in isoforms SPCA1a, SPCA1b, and SPCA1d, in the cytosolic tail immediately after the M10 (Figure 4c) in a region that is predicted to be a helical extension of M10 in all three isoforms (compare Figures $4 \mathrm{a}$ and b). This motif is also highly conserved throughout different species suggesting functional importance (Figure 4d).

Therefore, SPCA1 is potentially involved in the interaction with COPI. This reinforces the idea that SPCA1 could be involved in regulating local fusion/fission of cargo domain/ vesicles carrying proteins along the secretory pathway, triggering the cytosolic $\mathrm{Ca}^{2+}$ level in the peri-golgi membranes, where $\mathrm{Ca}^{2+}$-binding proteins are temporarily recruited to organize and regulate the membrane fusion/fission, a fundamental process for cargo progression. ${ }^{4,57}$

The analysis of the potential of C-terminal tail residues to interact with protein partners also highlighted additional sequences, which are differentially present in the four isoforms (Figure 4c). In particular, the isoforms SPCA1b and SPCA1d both end with the C-terminal sequence EDVSCV, which is predicted to be a protein interaction site (Figure 4c). By inspecting the literature we realized that this motif perfectly reproduces the structural features that are necessary for recognition by class I PDZ domains: (i) being at the 
a

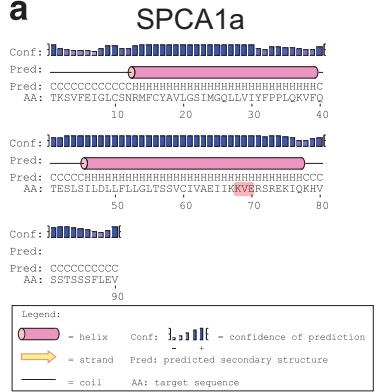

SPCA1b

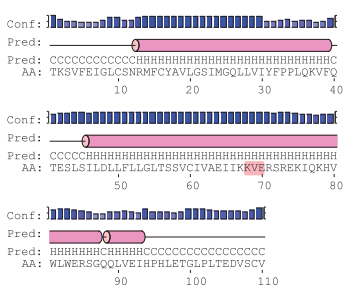

SPCA1C

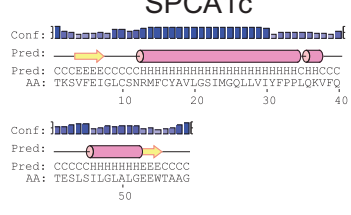

SPCA1d

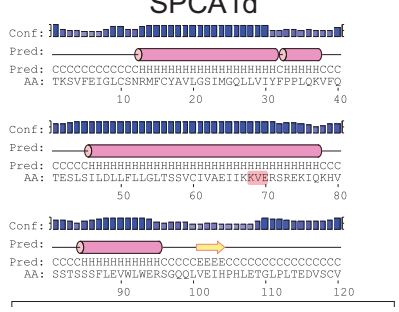

b
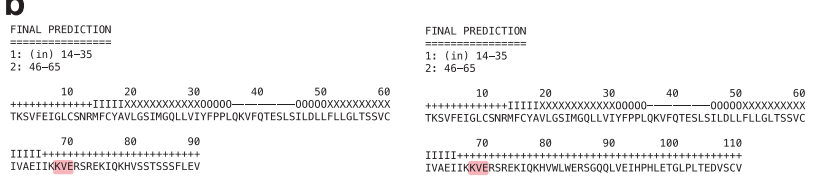

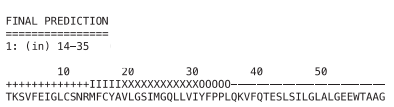

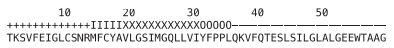

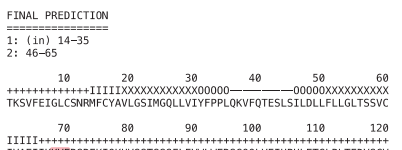

c

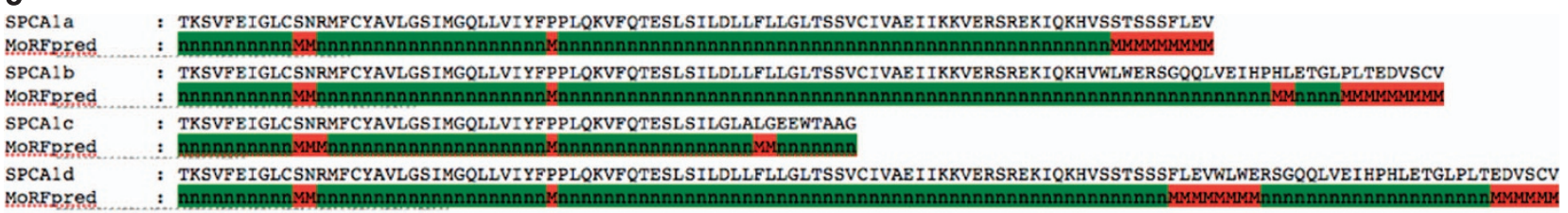

d

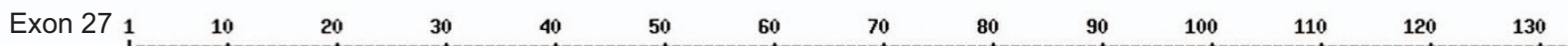

Homo sapiens

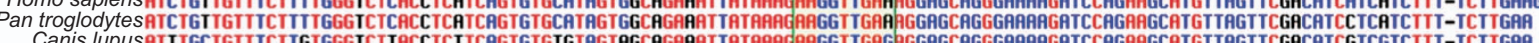

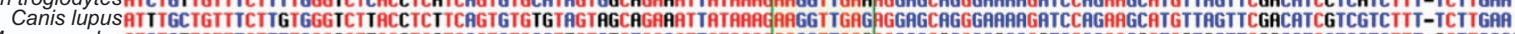

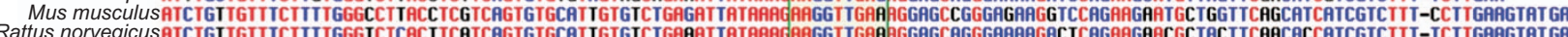

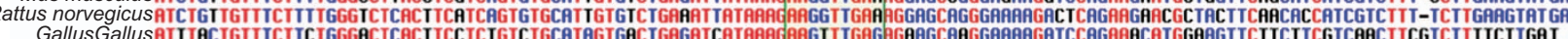

Gan

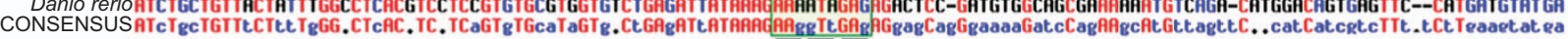

$\mathrm{K} \nabla \mathrm{E}$

Figure 4 Bioinformatics analysis of SPCA1 isoforms C-terminal region. (a) Secondary structure propensities in the proteins' regions encoded by terminal exons were predicted with the program PSIPRED. ${ }^{92}$ Highlighted in pink is the corresponding sequences in the KXD/E motif. Predictions indicate that this motif is located in a helical extension spanning outside transmembrane helix M10 (see also b). Interestingly this helix is predicted to have different length in the three isoforms where it is present (a, $\mathbf{b}$ and $\mathbf{d}$ ). (b) Transmembrane topology prediction was performed using the algorithms MEMSAT3 (ref. 93) and TMHMM2 (http://www.cbs.dtu.dk/services/TMHMM) yielding very similar results. In the isoforms SPCA1a, SPCA1b and SPCA1d, two transmembrane helices are predicted by both algorithms, the first (M9) with cytosolic-lumenal orientation and the second (M10) with opposite orientation. In the SPCA1c isoform a single cytosolic-lumenal transmembrane helix (M9) is predicted. Shown are results from MEMSAT3 where ' + ' stands for cytosolic residue, ' - ' for lumenal residue and I, X and O for transmembrane residues (I close to cytosolic, X central and O close to lumenal). (c) The MoRFPred algorithm ${ }^{94}$ was used to predict, in the different isoforms, regions of the protein that are prone to interact with protein partners, due to disorder-to-order transitions, here highlighted in red. The EDSCV motif found at the $\mathrm{C}$-terminal of isoforms $b$ and $d$ matches the consensus sequence specifically recognized by class I PDZ domains. The other regions predicted in isoforms 'a' and 'd' may be recognized by unknown protein partners. Highlighted in red are the putative residues of binding motifs. (d) The exon 27 encoding for most of the M10 domain and the C-terminal tail where putative binding motifs are located. The KXD/E is highly conserved. Sequence of Homo sapiens was compared to the four species of mammals chosen (Ensembl database. ID: Homo Sapiens ATP2C1-011 ENST00000428331; Pan troglodytes ATP2C1-201 ENSPTRT00000028742; Canis lupus ATP2C1-201 ENSCAFT00000031759; Mus musculus ATP2C1-005 ENSMUST00000038118; Rattus norvegicus ATP2C1-201 ENSRNOT00000018175) and two nonmammalian (Ensembl database. ID: Gallus gallus ATP2C1-201 ENSGALT00000018903; Danio rerio ATP2C1-001 ENSDART00000084528)

C-terminal of a protein and (ii) matching the consensus for the last three residues $\mathrm{S} / \mathrm{T}-X-\Phi-\mathrm{COO}-{ }^{58}$ where $X$ is any residue and $\Phi$ is a hydrophobic residue (often a valine). Thus, this motif is strongly predicted to interact with the PDZ domains of other proteins. ${ }^{59}$ Several proteins containing the PDZ domain can potentially interact with the tail of SPCA1b and SPCA1d modulating the function of the pump. Interestingly, evidences that the C-terminal tail of SPCA1 could interact with the golgi matrix arise from a recent observation of the interaction of C-terminal valine-bearing cargoes moving through the golgi apparatus by binding to GRASP55 and GRASP65, two golgi matrix proteins that contain PDZ domains. ${ }^{60}$ PDZ domain recognition of the C-terminal tail is involved in golgi maintenance and in cargo progression. ${ }^{60,61}$ The SPCA1 isoforms present a valine residue as last amino acid residue in the $\mathrm{C}$-terminal position (Figures 1c and 4c).

Also the FLEV motif present at the C-terminal end of SPCA1a and in the cytoplasmic tail of SPCA1d is predicted to be a protein binding motif (Figure $4 \mathrm{C}$ ). Interestingly, this motif is also present in rat calsequestrin (CASQ; AAA75480). CASQ regulates $\mathrm{Ca}^{2+}$-binding in the sarcoplasmic reticulum in 
cardiac muscle cells. However, this domain is not conserved between the human and murine proteins. This unique feature of the human protein could favor specific protein interactions by SPCA1 isoforms to mediate signaling and trigger the modulation of $\mathrm{Ca}^{2+}$ levels in the interested subcellular compartment. In the case of CASQ the interactors could be triadin and junctin. ${ }^{62}$ Analogs could have the same role in the golgi apparatus for SPCA1, working as $\mathrm{Ca}^{2+}$-mediated sensing probes to regulate the pump activity.

The above observations reinforce the hypothesis for a functional role of each different C-terminal tail in mediating interaction with different proteins.

\section{Importance of SPCA1 in Membrane Trafficking: What is the Possible Role of each Isoform?}

In addition to the maintenance of intra-golgi $\mathrm{Ca}^{2+}$ and $\mathrm{Mn}^{2+}$, which has direct effects on $\mathrm{Ca}^{2+}$-dependent proteases ${ }^{63}$ and $\mathrm{Mn}^{2+}$-dependent glycosyltransferases ${ }^{64}$ (see also below), there is also evidence that SPCA1 may affect cytosolic $\mathrm{Ca}^{2+}$ signaling. ${ }^{65-69} \mathrm{Ca}^{2+}$ released from the golgi leads to a localized increase in cytosolic $\mathrm{Ca}^{2+}$ that stimulates vesicle fusion, thus contributing to intra-golgi transport of cargo, ${ }^{70}$ consistent with the distribution of SPCA1 in the golgi apparatus. ${ }^{4}$ Thus, maintenance of luminal $\mathrm{Ca}^{2+}$ stores by SPCA1 may enable $\mathrm{Ca}^{2+}$ signaling events that directly affect the transport of cargocontaining domains from ER to the golgi apparatus and from there to their final destinations. ${ }^{3}$ If so, a deficiency in this signaling function likely contributes to the mislocalization of proteins in response to a decreased SPCA1 activity/level. ${ }^{71-73}$

Furthermore, effects on the ER were also observed in mammalian cells. Depletion of SPCA1 affected degradation of a mutant glycoproteins via ERAD, although it did not affect degradation of a non-glycoprotein substrates. ${ }^{71}$ Signaling pathways that mediate ER stress responses were intact and functional; however, SPCA1-deficient cells were highly sensitive to treatment with tunicamycin or thapsigargin, both of which cause ER stress and activate ER stress responses. ${ }^{71}$ Evidences of golgi stress, secondary effects on the ER, and stress responses were categorized as survival responses or apoptotic responses. ${ }^{72}$

Although the role of SPCA1 in the $\mathrm{Ca}^{2+}$ homeostasis of the golgi apparatus and its role in membrane trafficking has been well described and accepted, ${ }^{70,73}$ the role of single isoforms still needs to be investigated. Possible differential distribution along the secretory pathway at sub-organelle level as well as at tissue level remains completely uninvestigated too. SPCA1 isoforms differ from each other in their $\mathrm{C}$-terminal tails that is not directly involved in the regulation of $\mathrm{Ca}^{2+}$-binding. However, many $\mathrm{Ca}^{2+}$-binding proteins are recruited on the golgi membranes during the trafficking pulse (cPLA2, calmodulin, SNARE cofactors, etc.). ${ }^{3}$ These proteins are recruited on the golgi membranes through a local release of $\mathrm{Ca}^{2+}$ from the golgi apparatus and they should be released as soon as they fulfill their own function. ${ }^{3}$ The recruited $\mathrm{Ca}^{2+}$-binding proteins are addressed to the so-called lateral rims (or non compact zones) of the golgi stacks and in both the cisand trans-golgi area where SPCA1 is clearly localized. ${ }^{4}$ Whether SPCA1 C terminus tails are involved in establishing temporary contacts with $\mathrm{Ca}^{2+}$-binding recruited proteins remains unknown.

SPCA1 is also able to transport $\mathrm{Mn}^{2+}$ ions into the golgi with high affinity, an ability that SERCAs do not possess. As $\mathrm{Mn}^{2+}$ is not used for signaling like $\mathrm{Ca}^{2+}$, the main reason for removing it from the cytosol is to prevent $\mathrm{Mn}^{2+}$ toxicity. ${ }^{74}$ Several proteins within the pathway require either $\mathrm{Ca}^{2+}$ and $\mathrm{Mn}^{2+}$, or other divalent ions to function as metal cofactors, such as amino-peptidase $\mathrm{P}^{75}$ pro-protein convertases ${ }^{76}$ and sulfotransferarese. ${ }^{77}$ Even if there is no differential efficiency in the removal of ions by each SPCA1 isoform, potential differential sub-organellar localization of the isoforms may reflect the pump activity involved in different $\mathrm{Ca}^{2+} / \mathrm{Mn}^{2+}$-dependent pathways.

The inefficient removal of $\mathrm{Ca}^{2+} / \mathrm{Mn}^{2+}$ from the cytosol by SPCA1, with consequent decreased accumulation in the golgi, causes alteration in intracellular signaling and posttranscriptional protein modifications. $\mathrm{Mn}^{2+}$ is a fundamental cofactor of the golgi resident enzymes mannosidases. ${ }^{78}$ Reduced removal of $\mathrm{Ca}^{2+}$ from the cytosol during the arrival and the passage of cargo proteins to and through the golgi apparatus, significantly reduces physiological protein secretion. ${ }^{4}$

Furthermore, SPCA1 seems to have a direct role in organizing the cargo sorting at the trans-golgi network ${ }^{79,80}$ and subsequent departure. ${ }^{81}$ Functional SPCA1 is important for insulin-like growth factor receptor (IGF1R) processing in basal-like breast cancer, and inhibition of SPCA1 'may offer an alternative strategy to direct inhibitors of IGF1R and attenuate the processing of other proprotein convertase substrates.... ${ }^{82}$ However, which of the SPCA1 isoforms, if any, works at this level still needs to be defined.

\section{Membrane Trafficking in HHD Keratinocytes}

In the skin of HHD patients, one of the altered clusters of proteins affected by non-functional or depleted SPCA1 are the adhesion proteins. The loss of one functional copy of the ATP2C1 gene in HHD is characterized by the development of skin lesions and an associated loss of cell-cell adhesion. ${ }^{6,7}$ Proteins necessary for desmosome formation (as well as extracellular matrix components) are likely to be directly involved in cellular interactions and are affected in $\mathrm{SPCA} 1^{+/-}$ cells. ${ }^{83}$ Desmosomes are molecular complexes of cell adhesion proteins and linking proteins that attach the cell surface adhesion proteins to intracellular keratin cytoskeletal filaments. Desmoglein and desmocollin, two components of the desmosome, are members of the cadherin family of cell adhesion molecules. They are transmembrane proteins that bridge the space between adjacent epithelial cells by way of homophilic binding of their extracellular domains to other desmosomal cadherins on the adjacent cell. ${ }^{83}$ Both have five extracellular domains and have $\mathrm{Ca}^{2+}$-binding motifs. Defects in anchoring to cytoskeletal constituents can also reflect possible intracellular morphological defects as observed in golgi apparatus. ${ }^{84}$

Another important finding relating SPCA1 depletion and protein secretion in keratinocytes comes from a study on involucrin (a protein that makes up the cornified envelope of keratinocytes and is expressed in response to increased 
cytosolic $\left.\mathrm{Ca}^{2+}\right) \cdot{ }^{14}$ Involucrin is expressed at lower levels in cells from HHD patients or cells treated with SPCA1 RNAi when compared to normal keratinocytes. ${ }^{14}$ Decreased level of involucrin was caused by lower involucrin mRNA levels in HHD keratinocytes; decreased involucrin mRNA, in turn, was caused by increased rates of involucrin mRNA degradation. ${ }^{14}$ $\mathrm{Ca}^{2+}$-sensitive involucrin AP-1 promoter activity was increased, both in HHD keratinocytes and in RNAi experimental model, suggesting compensatory promoter up-regulation in the face of increased mRNA degradation. ${ }^{14}$ Thus, transport/secretion of desmosomes components are impaired by SPCA1 depletion.

Further observations revealed that claudins are also regulated by SPCA $1 .{ }^{85}$ Claudins are a family of proteins that are the most important components of the tight junctions, establishing a barrier that controls the flow of molecules in the intercellular space between the cells of an epithelium. In SPCA1-depleted keratinocytes protein levels of components of desmosomes, adherens and tight junctions did not show marked changes except for claudin1 and 4 that were increased. ${ }^{85}$

Whether and which SPCA1 isoforms are implicated in the regulation of specific cell adhesion protein trafficking is unknown. Nevertheless, the reason why desmosomes/tight junctions are affected more in the skin than in other epithelial tissues has to be investigated.

\section{Conclusions}

Distribution of SPCA1 isoforms along the secretory pathway in different cell types could lead to a better understanding of which isoform is doing what, when, and where. Considering that SPCA1 is ubiquitously expressed, the fundamental role of this protein in regulating the $\mathrm{Ca}^{2+} / \mathrm{Mn}^{2+}$ physiology ${ }^{86}$ and membrane trafficking in different cell types/tissues could be due to specific clusters of SPCA1 isoforms. Further investigations to clarify the role of each SPCA1 isoforms will benefit the understanding of the biology of secretion in health and disease, and will impact on several pathological conditions.

\section{Conflict of Interest}

The authors declare no conflict of interest.

Acknowledgements. Thanks to Dr Seetharaman Parashuraman, Institute of Protein Biochemistry, Naples, Italy for critical reading of the manuscript. This work was partly supported by a grant from the AIRC Associazione Italia Ricerca sul Cancro (IG-2014 15197 to LF).

1. De Matteis MA, Luini A. Exiting the golgi complex. Nat Rev Mol Cell Biol 2008; 9: 273-284.

2. Brandizzi $F$, Barlowe $C$. Organization of the ER-golgi interface for membrane traffic control. Nat Rev Mol Cell Biol 2013; 14: 382-392.

3. Micaroni M. Calcium around the golgi apparatus: implications for intracellular membrane trafficking. Adv Exp Med Biol 2012; 40: 439-460.

4. Micaroni M, Perinetti G, Berrie CP, Mironov AA. The SPCA1 $\mathrm{Ca}^{2+}$-pump and intracellular membrane trafficking. Traffic 2010; 11: 1315-1333.

5. Wootton LL, Argent CC, Wheatley M, Michelangeli F. The expression, activity and localisation of the secretory pathway Ca2+-ATPase (SPCA1) in different mammalian tissues. Biochim Biophys Acta 2004; 1664: 189-197.

6. Sudbrak R, Brown J, Dobson-Stone C, Carter S, Ramser J, White J et al. Hailey-Hailey disease is caused by mutations in ATP2C1 encoding a novel Ca2+ pump. Hum Mol Genet 2000; 9: 1131-1140.

7. Hu Z, Bonifas JM, Beech J, Bench G, Shigihara T, Ogawa $\mathrm{H}$ et al. Mutations in ATP2C1, encoding a calcium pump, cause Hailey-Hailey disease. Nat Genet 2000; 24: 61-65.
8. Matsuda M, Hamada T, Numata S, Teye K, Okazawa H, Imafuku S et al. Mutationdependent effects on mRNA and protein expressions in cultured keratinocytes of HaileyHailey disease. Exp Dermatol 2014; 23: 509-528.

9. Hailey $\mathrm{H}$, Hailey $\mathrm{H}$. Familial benign chronic pemphigus. Report of 13 cases in four generations of a family and report of 9 additional cases in 4 generations of a family. Archiv Dermatol Syphilol 1939; 39: 679-685.

10. James W, Berger T, Elston D. Andrews' Diseases of the Skin: Clinical Dermatology, 10th edn. Saunders: Philadelphia, PA, 2005.

11. Freedberg IM, Goldsmith LA, Katz S, Austen F, Wolff K. Fitzpatrick's Dermatology in General Medicine, 6th edn. McGraw-Hill: New York, NY, 2003.

12. Foggia $L$, Hovnanian A. Calcium pump disorders of the skin. Am J Med Genet $C$ Semin Med Genet 2004; 131C: 20-31.

13. Dobson-Stone C, Fairclough R, Dunne E, Brown J, Dissanayake M, Munro CS et al. Hailey-Hailey disease. molecular and clinical characterization of novel mutations in the ATP2C1 gene. J Invest Dermatol 2002; 118: 338-343.

14. Aberg KM, Racz E, Behne MJ, Mauro TM. Involucrin expression is decreased in HaileyHailey keratinocytes owing to increased involucrin mRNA degradation. J Invest Dermatol 2007; 127: 1973-1979.

15. Bergman R, Levy R, Pam Z, Lichtig C, Hazaz B, Friedman-Birnbaum R. A study of keratin expression in benign familial chronic pemphigus. Am J Dermatopathol 1992; 14: 32-36.

16. Su MJ, Bikle DD, Mancianti ML, Pillai S. 1,25-Dihydroxyvitamin D3 potentiates the keratinocyte response to calcium. J Biol Chem 1994; 269: 14723-14729.

17. Rice WJ, MacLennan DH. Scanning mutagenesis reveals a similar pattern of mutation sensitivity in transmembrane sequences $M 4, M 5$, and $M 6$, but not in $\mathrm{M} 8$, of the $\mathrm{Ca} 2$ +-ATPase of sarcoplasmic reticulum (SERCA1a). J Biol Chem 1996; 271: 31412-31419.

18. Chao SC, Tsai YM, Yang MH. Mutation analysis of ATP2C1 gene in Taiwanese patients with Hailey-Hailey disease. Br J Dermatol 2002; 146: 595-600.

19. Btadini W, Abou Hassan KO, Saadeh D, Abbas O, Ballout F, Kibbi AG et al. Identification of several mutations in ATP2C1 in Lebanese families: insight into the pathogenesis of HaileyHailey disease. PLoS One 2015; 10: e0115530.

20. Ikeda S, Shigihara T, Mayuzumi N, Yu X, Ogawa H. Mutations of ATP2C1 in Japanese patients with Hailey-Hailey disease: intrafamilial and interfamilial phenotype variations and lack of correlation with mutation patterns. J Invest Dermatol 2001; 117: 1654-1656.

21. Yokota K, Takizawa Y, Yasukawa K, Kimura K, Nishikawa T, Shimizu H. Analysis of ATP2C1 gene mutation in 10 unrelated Japanese families with Hailey-Hailey disease. J Invest Dermatol 2002; 118: 550-551.

22. Li H, Sun HK, Zhu XJ. Four novel mutations in ATP2C1 found in Chinese patients with Hailey-Hailey disease. Br J Dermatol 2003; 149: 471-474.

23. Fairclough RJ, Lonie L, Van Baelen K, Haftek M, Munro CS, Burge SM et al. Hailey-Hailey disease: identification of novel mutations in ATP2C 1 and effect of missense mutation A528P on protein expression levels. J Invest Dermatol 2004; 123: 67-71.

24. Hamada T, Fukuda S, Sakaguchi S, Yasumoto S, Kim SC, Hashimoto T. Molecular and clinical characterization in Japanese and Korean patients with Hailey-Hailey disease: six new mutations in the ATP2C1 gene. J Dermatol Sci 2008; 51: 31-36.

25. Cialfi S, Oliviero C, Ceccarelli S, Marchese C, Barbieri L, Biolcati G et al. Complex multipathways alterations and oxidative stress are associated with Hailey-Hailey disease. Br J Dermatol 2010; 162: 518-526.

26. Szigeti R, Chao SC, Várszegi D, Czakó M, Kosztolányi G, Kellermayer R. The first genetically supported case of chronic benign pemphigus Hailey-Hailey disease in Hungary. Orv Hetil 2005; 146: 1933-1935.

27. Kellermayer R, Szigeti R, Keeling KM, Bedekovics T, Bedwell DM. Aminoglycosides as potential pharmacogenetic agents in the treatment of Hailey-Hailey disease. $J$ Invest Dermatol 2006; 126: 229-231.

28. Cheng TS, Ho KM, Lam CW. Heterogeneous mutations of the ATP2C1 gene causing Hailey-Hailey disease in Hong Kong Chinese. J Eur Acad Dermatol Venereol 2010; 24: 1202-1206.

29. Racz E, Csikos M, Karpati S. Novel mutations in the ATP2C1 gene in two patients with Hailey-Hailey disease. Clin Exp Dermatol 2005; 30: 575-577.

30. Zhang $X Q$, Wu HZ, Li BX, Xu YS, Wu JB, Lin LL et al. Mutations in the ATP2C1 gene in Chinese patients with Hailey-Hailey disease. Clin Exp Dermatol 2006; 31: 702-705.

31. Zhang HZ, Tian HQ, Du DH, Wang GJ, Yan XX, Liu H et al. Analysis of ATP2C1 gene mutations in Chinese patients with Hailey-Hailey disease. Clin Exp Dermatol 2012; 37: 190-193.

32. Zhang D, Li X, Xiao S, Huo J, Wang S, Zhou P. Detection and comparison of two types of ATP2C1 gene mutations in Chinese patients with Hailey-Hailey disease. Arch Dermatol Res 2012; 304: 163-170.

33. Majore S, Biolcati G, Barboni L, Cannistraci C, Binni F, Crisi A et al. ATP2C1 gene mutation analysis in Italian patients with Hailey-Hailey disease. J Invest Dermatol 2005; 125: 933-935.

34. Nemoto-Hasebe I, Akiyama M, Osawa R, Nakamura H, Shimizu H. Diagnosis of HaileyHailey disease facilitated by DNA testing: a novel mutation of ATP2C1. Acta Derm Venereol 2008; 88: 399-400.

35. Kong A, Frigge ML, Masson G, Besenbacher S, Sulem P, Magnusson G et al. Rate of de novo mutations and the importance of father's age to disease risk. Nature 2012; 488 : 471-475.

36. Fairclough RJ, Dode L, Vanoevelen J, Andersen JP, Missiaen L, Raeymaekers L et al. Effect of Hailey-Hailey disease mutations on the function of a new variant of human secretory pathway Ca2+/Mn2+-ATPase (hSPCA1). J Biol Chem 2003; 278: 24721-24730. 
37. Dode L, Andersen JP, Raeymaekers, Missiaen L, Vilsen B, Wuytack F. Functional comparison between secretory pathway $\mathrm{Ca}^{2+} / \mathrm{Mn}^{2+}$-ATPase (SPCA) 1 and sarcoplasmic reticulum $\mathrm{Ca}^{2+}$-ATPase (SERCA) 1 isoforms by steady-state and transient kinetic analyses. J Biol Chem 2005; 280: 39124-39134.

38. Zhang D, Li X, Wang Z, Zhang Y, Guo K, Wang S et al. Hailey-Hailey disease: investigation of a possible compensatory SERCA2 up-regulation and analysis of SPCA1, p63, and IRF6 expression. Arch Dermatol Res 2015; 307: 143-149.

39. Missiaen L, Dode L, Vanoevelen J, Raeymaekers L, Wuytack F. Calcium in the golgi apparatus. Cell Calcium 2007; 41: 405-416.

40. Mukhopadhyay S, Linstedt AD. Identification of a gain-of-function mutation in a golgi P-type ATPase that enhances Mn2+ efflux and protects against toxicity. Proc Natl Acad Sci USA 2011; 108: 858-863.

41. Shull GE, Okunade G, Liu LH, Kozel P, Periasamy M, Lorenz JN et al. Physiological function of plasma membrane and intracellular $\mathrm{Ca}^{2+}$ pumps revealed by analysis of null mutants. Ann NY Acad Sci 2003; 986: 453-460.

42. Mandal D, Woolf TB, Rao R. Manganese selectivity of pmr1, the yeast secretory pathway ion pump, is defined by residue Gln783 in transmembrane segment 6. Residue Asp778 is essential for cation transport. J Biol Chem 2000; 275: 23933-23938.

43. Mandal $D$, Rulli SJ, Rao R. Packing interactions between transmembrane helices alter ion selectivity of the yeast golgi $\mathrm{Ca}^{2+} / \mathrm{Mn}^{2+}$-ATPase PMR1. J Biol Chem 2003; 278: 35292-35298.

44. Kinzle C, Basnet N, Crevenna AH, Beck G, Habermann B, Mizuno N et al. Cofilin recruits F-actin to SPCA1 and promotes $\mathrm{Ca}^{2+}$-mediated secretory cargo sorting. J Cell Biol 2014; 206: 635-654.

45. Chun SI, Whang KC, Su WP. Squamous cell carcinoma arising in Hailey-Hailey disease. $J$ Cutan Pathol 1988; 5: 234-237.

46. Cockayne SE, RassI DM, Thomas SE. Squamous cell carcinoma arising in Hailey-Hailey disease of the vulva. Br J Dermatol 2000; 142: 540-542

47. Holst VA, Fair KP, Wilson BG, Patterson JW. Squamous cell carcinoma arising in HaileyHailey disease. J Am Acad Dermatol 2000; 43: 368-371.

48. Okunade GW, Miller ML, Azhar M, Andringa A, Sanford LP, Doetschman T et al. Loss of the Atp2c1 secretory pathway $\mathrm{Ca}(2+)$-ATPase (SPCA1) in mice causes golgi stress, apoptosis, and midgestational death in homozygous embryos and squamous cell tumors in adult heterozygotes. J Biol Chem 2007; 282: 26517-26527.

49. Shull GE, Miller ML, Prasad V. Secretory pathway stress responses as possible mechanisms of disease involving golgi $\mathrm{Ca}^{2+}$ pump dysfunction. Biofactors 2011; 37: 150-158.

50. Micaroni M, Malquori L. Overlapping ATP2C1 and ASTE1 genes in human genome: implications for SPCA1 isoforms expression? Int J Mol Sci 2013; 14: 674-683.

51. Cosson P, Letourneur F. Coatomer interaction with di-lysine endoplasmic reticulum retention motifs. Science 1994; 263: 1629-1631.

52. Letourneur F, Gaynor EC, Hennecke S, Demolliere C, Duden R, Emr SD et al. Coatomer is essential for retrieval of dilysine-tagged proteins to the endoplasmic-reticulum. Cell 1994; 79: 1199-1207.

53. Contreras I, Yang Y, Robinson DG, Aniento F. Sorting signals in the cytosolic tail of plant p24 proteins involved in the interaction with the COPII coat. Plant Cell Physiol 2004; 45: 1779-1786

54. Tu L, Tai WC, Chen L, Banfield DK. Signal-mediated dynamic retention of glycosyltransferases in the golgi. Science 2008; 321: 404-407.

55. Gao G, Cai Y, Wang Y, Kang BH, Aniento F, Robinson DG et al. Retention mechanisms for ER and golgi membrane proteins. Trend Plant Sci 2014; 19: 508-515.

56. Woo CH, Gao C, Yu P, Tu L, Meng Z, Banfield DK et al. Conserved function of the lysinebased $K X D / E$ motif in golgi retention for endomembrane proteins among different organisms. Mol Biol Cell 2015; 26: 4280-4293.

57. Micaroni M. The role of calcium in intracellular trafficking. Curr Mol Med 2010; 10: 763-773.

58. Hung AY, Sheng M. PDZ domains: structural modules for protein complex assembly. J Biol Chem 2002; 277: 5699-5702.

59. Jemth $P$, Gianni S. PDZ domains: folding and binding. Biochemestry 2007; 46: 8701-8708.

60. D'Angelo G, Prencipe L, lodice L, Beznoussenko G, Savarese M, Marra P et al. GRASP65 and GRASP55 sequentially promote the transport of C-terminal valine-bearing cargos to and through the golgi complex. J Biol Chem 2009; 284: 34849-34860.

61. Barr FA, Puype M, Vandekerckhove J, Warren G. GRASP65, a protein involved in the stacking of golgi cisternae. Cell 1997; 91: 253-262.

62. Zhang L, Kelley J, Schmeisser G, Kobayashi YM, Jones LR. Complex formation between junctin, triadin, calsequestrin, and the ryanodine receptor. Proteins of the cardiac junctional sarcoplasmic reticulum membrane. J Biol Chem 1997; 272: 23389-23397.

63. Antebi A, Fink GR. The yeast $\mathrm{Ca}^{2+}$-ATPase homologue, PMR1, is required for normal golgi function and localizes in a novel golgi-like distribution. Mol Biol Cell 1992; 3: 633-654.

64. Varki A. Factors controlling the glycosylation potential of the golgi apparatus. Trends Cell Biol 1998; 8: 34-40.

65. Callewaert G, Parys JB, De Smedt H, Raeymaekers L, Wuytack F, Vanoevelen J et al. Similar $\mathrm{Ca}^{2+}$-signaling properties in keratinocytes and in COS-1 cells overexpressing the secretory-pathway Ca ${ }^{2+}$-ATPase SPCA1. Cell Calcium 2003; 34: 157-162.

66. Harper C, Wootton L, Michelangeli F, Lefièvre L, Barratt C, Publicover S. Secretory pathway $\mathrm{Ca}^{2+}$-ATPase (SPCA1) $\mathrm{Ca}^{2+}$-pumps, not SERCAs, regulate complex $\left[\mathrm{Ca}^{2+}\right]_{\mathrm{i}}$ signals in human spermatozoa. J Cell Sci 2005; 118: 1673-1685.
67. Foggia L, Aronchik I, Aberg K, Brown B, Hovnanian A, Mauro TM. Activity of the hSPCA1 golgi $\mathrm{Ca}^{2+}$-pump is essential for $\mathrm{Ca}^{2+}$-mediated $\mathrm{Ca}^{2+}$ response and cell viability in Darier disease. J Cell Sci 2006; 119: 671-679.

68. Baron S, Struyf S, Wuytack F, Van Damme J, Missiaen L, Raeymaekers L et al Contribution of intracellular $\mathrm{Ca}^{2+}$ stores to $\mathrm{Ca}^{2+}$ signaling during chemokinesis of human neutrophil granulocytes. Biochim Biophys Acta 2009; 1793: 1041-1049.

69. Lai $P$, Michelangeli $F$. Changes in expression and activity of the secretory pathway $\mathrm{Ca}^{2+}$ ATPase 1 (SPCA1) in A7r5 vascular smooth muscle cells cultured at different glucose concentrations. Biosci Rep 2009; 29: 397-404.

70. Micaroni M, Perinetti G, Di Giandomenico D, Bianchi K, Spaar A, Mironov AA. Synchronous intra-golgi transport induces release of $\mathrm{Ca}^{2+}$-from the golgi apparatus. Exp Cell Res 2010; 316: 2071-2086.

71. Ramos-Castañeda J, Park YN, Liu M, Hauser K, Rudolph H, Shull GE et al. Deficiency of ATP2C1, a golgi ion pump, induces secretory pathway defects in endoplasmic reticulum (ER)-associated degradation and sensitivity to ER stress. J Biol Chem 2005; 280: 9467-9473.

72. Sepúlveda MR, Vanoevelen J, Raeymaekers L, Mata AM, Wuytack F. Silencing the SPCA1 (secretory pathway $\mathrm{Ca}^{2+}$-ATPase isoform 1) impairs $\mathrm{Ca}^{2+}$ homeostasis in the golgi and disturbs neural polarity. J Neurosci 2009; 29: 12174-12182.

73. Lissandron V, Podini P, Pizzo P, Pozzan T. Unique characteristics of $\mathrm{Ca}^{2+}$ homeostasis of the trans-golgi compartment. Proc Natl Acad Sci USA 2010; 107: 9198-9203.

74. Van Baelen K, Dode L, Vanoevelen J, Callewaert G, De Smedt H, Missiaen L et al. The $\mathrm{Ca} 2+/ \mathrm{Mn} 2+$ pumps in the golgi apparatus. Biochim Biophys Acta 2004; 1742: 103-112.

75. Cottrell GS, Hooper NM, Turner AJ. Cloning, expression, and characterization of human cytosolic aminopeptidase P: a single manganese(II)-dependent enzyme. Biochemistry 2000; 39: 15121-15128.

76. Davidson HW, Rhodes CJ, Hutton JC. Intraorganellar calcium and pH control proinsulin cleavage in the pancreatic beta cell via two distinct site-specific endopeptidases. Nature 1988; 333: 93-96.

77. Mishiro E, Liu MY, Sakakibara Y, Suizo M, Liu MC. Zebrafish tyrosylprotein sulfotransferase: molecular cloning, expression, and functional characterization. Biochem Cell Biol 2004; 82: 295-303.

78. Tkacz JS, Herscovics A, Warren CD, Jeanloz RW. Mannosyltransferase activity in calf pancreas microsomes. Formation from guanosine diphosphate-D- $\left({ }^{14} \mathrm{C}\right)$ mannose of a ${ }^{14} \mathrm{C}$ labeled mannolipid with properties of dolichyl mannopyranosyl phosphate. J Biol Chem 1974; 249: 6372-6381

79. Curwin AJ, von Blume J, Malhotra V. Cofilin-mediated sorting and export of specific cargo from the golgi apparatus in yeast. Mol Biol Cell 2012; 23: 2327-2338.

80. von Blume J, Alleaume AM, Kienzle C, Carreras-Sureda A, Valverde M, Malhotra V. Cab45 is required for $\mathrm{Ca}(2+)$-dependent secretory cargo sorting at the trans-golgi network. J Cell Biol 2012; 199: 1057-1066

81. von Blume J, Alleaume AM, Cantero-Recasens G, Curwin A, Carreras-Sureda A, Zimmermann $T$ et al. ADF/cofilin regulates secretory cargo sorting at the TGN via the $\mathrm{Ca2+}$ ATPase SPCA1. Dev Cell 2011; 20: 652-662.

82. Grice DM, Vetter I, Faddy HM, Kenny PA, Roberts-Thomson SJ, Monteith GR. Golgi calcium pump secretory pathway calcium ATPase 1 (SPCA1) is a key regulator of insulinlike growth factor receptor (IGF1R) processing in the basal-like breast cancer cell line MDAMB-231. J Biol Chem 2010; 285: 37458-37466.

83. Delva E, Tucker DK, Kowalczyk AP. The desmosome. Cold Spring Harb Perspect Biol 2009; 1: a002543

84. Bornslaeger EA, Corcoran CM, Stappenbeck TS, Green KJ. Breaking the connection: displacement of the desmosomal plaque protein desmoplakin from cell-cell interfaces disrupts anchorage of intermediate filament bundles and alters intercellular junction assembly. J Cell Biol 1996; 134: 985-1001.

85. Raiko L, Siljamäki E, Mahoney MG, Putaala H, Suominen E, Peltonen J et al. Hailey-Hailey disease and tight junctions: Claudins 1 and 4 are regulated by ATP2C 1 gene encoding $\mathrm{Ca}(2+) / \mathrm{Mn}(2+)$ ATPase SPCA1 in cultured keratinocytes. Exp Dermatol 2012; 21: 586-591.

86. Brini M, Carafoli E. Calcium pumps in health and disease. Physiol Rev 2009; 89 : 1341-1378.

87. Luo S, Ni H, Li Y, Hou S, Li X, Liu Q. Novel clinical and molecular findings in Chinese families with Hailey-Hailey disease. Clin Exp Dermatol 2011; 36: 814-816.

88. Micaroni M. Correct reading frame helps to properly identify ATP2C1 gene mutations vs polymorphisms. Clin Exp Dermatol 2016 (doi:10.1111/ced.12836).

89. Li H, Sun X, Bu D, Zhu X. Study on gene mutation in 11 chinese families with Hailey-Hailey disease. Beijing Da Xue Xue Bao 2003; 35: 390-393.

90. Micaroni M. Misinterpretation of ATP2C1 mutations. Indian J Dermatol Venereol Leprol 2016; 82: 306-307.

91. Chao SC, Lee JYY, Wu MC, Hsu MML. A novel splice mutation in the ATP2C1 gene in a woman with concomitant psoriasis vulgaris and disseminated Hailey-Hailey disease. Int $J$ Dermatol 2012; 51: 947-951.

92. Jones DT. Protein secondary structure prediction based on position-specific scoring matrices. J Mol Biol 1999; 292: 195-202.

93. Nugent T, Jones DT. Transmembrane protein topology predictions using support vector machines. BMC Bioinformatics 2009; 10: 159.

94. Disfani FM, Hsu WL, Mizianty MJ, Oldfield CJ, Xue B, Dunker AK et al. MoRFpred, a computational tool for sequence-based prediction and characterization of short disorder-toorder transitioning binding regions in proteins. Bioinformatics 2012; 28: i75-i83. 
95. Zhang ZZ, Liang YH, Quan C, Gao M, Xiao FL, Yang S et al. Three novel ATP2C1 mutations in Chinese patients with Hailey-Hailey disease. Br J Dermatol 2008; 158 831-833.

96. Li X, Zhang D, Xiao S, Peng Z. Four novel mutations of the ATP2C1 gene in Chinese patients are associated with familial binign chronic pemphigus. Clin Exp Dermatol 2012; 37 797-799.

97. Tian H, Chen M, You J, Fu X, Liu H, Shi Z et al. Six novel mutations of ATP2C1 identified in eight Chinese patients with Hailey-Hailey disease. Indian J Dermatol Venereol Leprol 2013; 79: 245-247.

98. Shi BJ, Xiao S, Zhang Z, Lu J, Xue M, Jiang Y et al. The ATP2C1 gene in Hailey-Hailey disease patients: one novel deletion and one novel splicing mutation. J Eur Acad Dermatol Venereol 2015; 29: 2495-2497.

99. Zhang GL, Sun YT, Shi HJ, Gu Y, Shao MH, Du XF. Mutation analysis of ATP2C1 gene in a Chinese family with Hailey-Hailey disease. Zhonghua Yi Xue Yi Chuan Xue Za Zhi 2010; 27: 414-416

100. Shibata A, Sugiura K, Kimura U, Takamori K, Akiyama M. A novel ATP2C1 early truncation mutation suggests haploinsufficiency as a pathogenic mechanism in a patient with HaileyHailey disease. Acta Derm Venereol 2013; 93: 719-720.

101. Zhang ZZ, Li W, Zhou FS, Gao M, Xiao FL, Fang QY et al. Identification of a novel mutation in the ATP2C1 gene in a Chinese pedigree with Hailey-Hailey disease. Zhongguo Yi Xue Ke Xue Yuan Xue Bao 2007; 29: 163-166.

102. Zhang ZZ, Quan $\mathrm{C}, \mathrm{Mu} Y Z$, Yang $\mathrm{H}$ et al. Study on the mutations of ATP2C1 gene in six cases with familial benign chronic pemphigus. Chin J Dermatol 2014; 28: 441-443.

103. Pernet C, Bessis D, Savignac M, Tron E, Guillot B, Hovnanian A. Genitoperineal popular acantholytic dysckeratosis is allelic to Hailey-Hailey disease. Br J Dermatol 2012; 167 210-212.

104. Zhang F, Yan X, Jiang D, Tian H, Wang C, Yu L. Eight novel mutations of ATP2C1 identified in 17 Chinese families with Hailey-Hailey disease. Dermatology 2007; 215: 277-283.

105. Tian H, Yan X, Liu H, Yu Y, Zhang F. Six novel mutations identified in Chinese patients with Hailey-Hailey disease. J Dermatol Sci 2010; 58: 80-82.

106. Chang $\mathrm{HQ}$, Xiao FL, Zhou FS, Fang QY et al. Mutation detection of the ATP2C1 gene in a Chinese family with Hailey-Hailey disease. Acta Univer Med Anhui 2008; 43: 212-214.

107. Meng L, Gu Y, Du XF, Shao MH, Zhang LL, Zhang GL et al. Two novel ATP2C1 mutations in patients with Hailey-Hailey disease and a literature review of sequence variants reported in Chinese population. Genet Mol Res 2015; 14: 19349-19359.

108. Xu Z, Zhang L, Xiao Y, Li L, Lin Z, Yang Y et al. A case of Hailey-Hailey disease in an infant with a new ATP2C1 gene mutation. Pediatric Dermatol 2011; 28: 165-168.

109. lijima S, Hamada T, Kanzaki M, Ohata C, Hashimoto T. Sibling cases of Hailey-Hailey disease showing atypical clinical features and unique disease course. JAMA Dermatol 2014; 150: 97-99.

110. Xing XS, Wang Z, Liu S, Wu YH, Zhang SF, Wang SS et al. Three novel mutations of the ATP2C1 gene in Chinese families with Hailey-Hailey disease. J Eur Acad Dermatol Venereol 2016; 30: 1057-1059.

111. Ma YM, Zhang XJ, Liang YH, Ma L, Sun LD, Zhou FS et al. Genetic diagnosis in a Chinese Hailey-Hailey disease pedigree with novel ATP2C1 mutation. Arch Dermatol Res 2008; 300: 203-207.

112. Cheng YU, Cheng YM, Zhao G, Jia MC. A novel missense mutation of the ATP2C1 gene in a Chinese patient with Hailey-Hailey disease. Biochem Biophys Res Commun 2011; 406 420-422.

113. Ding TG, Fang H, Lao LM, Jiang XJ, Chen HC. Genetic diagnosis of Hailey-Hailey disease in two Chinese families: novel mutations in the ATP2C1 gene. Clin Exp Dermatol 2009; 34: e968-e971.

114. Ohtsuka T, Okita H, Hama N, Yamazaki S. Novel mutation of ATP2C1 gene in a Japanese patient with Hailey-Hailey disease. Dermatology 2006; 212: 194-197.

115. Matsuda M, Hamada T, Numata S, Teye K, Ishii N, Ohata $C$ et al. A three-dimensional in vitro culture model of Hailey-Hailey disease. Exp Dermatol 2015; 24: 788-789.
116. Wang CC, Chao SC, Tsai TH. Hailey-Hailey disease: a novel mutation of the ATP2C1 gene in a Taiwanese family with divergent clinical presentation. J Eur Acad Dermatol Venereol 2008; 22: 1145-1146.

117. Xu QQ, Cheng CZ, Huo J, Liu Y et al. Detection of ATP2C1 gene mutations in two Chinese families with Hailey-Hailey disease. Chin J Dermatol 2012; 26: 475-476.

118. Li XL, Peng ZH, Xiao SX, Wang ZH, Liu Y, Pan M et al. A novel deletion mutation of the ATP2C1 gene in Chinese patients with Hailey-Hailey disease. J Eur Acad Dermatol Venereol 2008; 22: 235-262.

119. Yokota K, Sawamura D. Hailey-Hailey disease with affective disorder: report of a case with novel ATP2C1 gene mutation. J Dermatol Sci 2006; 43: 150-151.

120. Szigeti R, Chao SC, Szász O, Tészás A, Kosztolányi G, Kellermayer R. Premenstrual exacerbation in calcium ATPase disorders of the skin. J Eur Acad Dermatol Venereol 2007; 21: 412-413.

121. Mizuno $\mathrm{K}$, Hamada $\mathrm{T}$, Hashimoto $\mathrm{T}$, Okamoto $\mathrm{H}$. Successful treatment with narrow-band UVB therapy for a case of generalized Hailey-Hailey disease with a novel splice-site mutation in ATP2C1 gene. Dermatol Ther 2014; 27: 233-235.

122. Zhu YG, Yang S, Gao M, Chen JJ, Li W, Wang PG et al. Two novel mutations of the ATP2C1 gene in Chinese families with Hailey-Hailey disease. J Dermatol Sci 2006; 42 : 125-127.

123. Yin $\mathrm{CH}$, Wang BX, Ma DL. Gene mutations in a Chinese family with Hailey-Hailey disease. Chin J Dermatol 2003; 36: 507-509.

124. Li X, Xiao S, Peng Z, Liu Y, Wang J, Zhou X. Two novel mutations of the ATP2C1 gene in Chinese patients with Hailey-Hailey disease. Arch Dermatol Res 2007; 299: 209-211.

125. Poblete-Gutiérrez $P$, Wiederholt $T$, König $A$, Jugert FK, Marquardt $Y$, Rübben $A$ et al. Allelic loss underlies type 2 segmental Hailey-Hailey disease, providing molecular confirmation of a novel genetic concept. J Clin Invest 2004; 114: 1467-1474.

126. Liu JZ, Yang T, Li X, Liu M, Wang QK, Liu JY. A novel mutation in the ATP2C1 gene is associated with Hailey-Hailey disease in a Chinese family. Int J Dermatol 2009; 48: 47-51.

127. Lipoff JB, Mudgil AV, Young S, Chu P, Cohen SR. Acantholytic dermatosis of the crural folds with ATP2C1 mutation is a possible variant of Hailey-Hailey disease. J Cutan Med Surg 2009; 13: 151-154

128. Chen S, Huang C, Li J. Detection of ATP2C1 gene mutation in familial benign chronic pemphigus. J Huazhong Univ Sci Technolog Med Sci 2005; 25: 585-586.

129. Van Beek N, Patsatsi A, Gupta Y, Moller S, Freitag M, Lemcke S et al. A family with atypical Hailey-Hailey disease-is there more to the underlying genetics than ATP2C1? PLOS One 2015; 10: e121253.

130. Chen SY, Huang CZ, Li JW. Mutation detection of ATP2C1 gene in a Chinese family with Hailey-Hailey disease. Chin J Dermatol 2004; 37: 550.

131. Makino T, Shimizu K, Mizawa M, Nakano H, Sawamura D, Shimizu T. A novel deletion mutation of the ATP2C1 gene in a family with Hailey-Hailey disease. Eur J Dermatol 2015 (doi:10.1684/ejd.2015.2592)

(c) (i) Cell Death and Disease is an open-access journal published by Nature Publishing Group. This work is licensed under a Creative Commons Attribution 4.0 International License. The images or other third party material in this article are included in the article's Creative Commons license, unless indicated otherwise in the credit line; if the material is not included under the Creative Commons license, users will need to obtain permission from the license holder to reproduce the material. To view a copy of this license, visit http://creativecommons.org/licenses/by/4.0/ 